de la viande et du lait, un anticle sur l'approvisionnement des villes, en lait, dans lequel il disait que G. Masno, à Milan, avait élaboré un programme pour la municipalisation du lait. Plus tand, Masvo a écrit, sur cette forme de l'approvisionnement en lait, un livwe intitulé : l'Approvigionnemento del Latte. Ce système; qui était très bien conçu, n'a pu être mis en pratique: Masca a neeonnu lui-même que son système n'était pas réalisable et, malheureusement, son travail de presque vingt années a été vain.

Je donne eneore ei-contre, p. 366, un bref exposé sur le nombre d'habitants dans quelques grandes villes en Allemagne età l'Etranger, sur la consommation du lait, sur son prix. Ces chiffres répondent aux indications qui m'ont été envoyées de l'Etranger par des experts en matière de lait dont la plupart me sont connus déjà depuis vingt ans. Une partie des éléments que j'ai recueillis en Allemagne, m'a été envoyée par des experts ; l'autre partie, par les administrations, en réponse à mes questions.

Pour terminer, c'est pour moi un agréable devoir de remercier tous ceux qui m'ont aidé dans mon travail, par l'envoi des éléments qui m'étaient nécessaires pour le mener à bonne fin.

\title{
LE PROCÈS DE LA MATIĖRE GRASSE DU LAIT
} par Ch. PORCHER

- sUITE -

Tous les faits dont il vient d'être parlé posent quelques questions auxquelles nous-devons répondre.

La cause de l'enrichissement du lait en matière grasse du commencement à la fin de la traite. - On doit d'abord se demander pourquoi le lait s'enrichit ainsi, plus ou moins régulièrement, mais d'une façon très nette, du commencement à la fin de la traite. On ne saurait invoquer, comme SANSon et plusieurs autres l'ont fait autrefois, la différence de densité entre la matière grasse et la partie aqueuse du lait et de laquelle résulterait l'ascension des globules graisseux dans les parties supérieures des canaux galactifères.

Pour Kirchner (Handbuch der Milchwirtschaft, 1888), les globules sont retenus mécaniquement dans les fins canaux de la glande et e'est dans le dernien lait trait qu'ils passent en plus grande quantité. Ils se collent contre les parois et en sont détzohés petit à petit par le flux lacté appelé par la mulsion. Si, au courant centrifuge en ques+ tion, on ajoute le fait d'agiter, de soubattre la glande, et dont l'influence s'aceroît au fur et à mesure que la traite s'avance, on conçoit 
que les laits s'enrichissent du commencement à la fin, une quantité de plus en plus grande de globules se trouvant détachés des parois des canaux par la continuité et l'exagération des causes qui ont commencé à en produire le décollement.

Il est d'ailleurs fort difficile de recueillir toute la graisse, et la mamelle d'une vache, tuée de suite après une traite poussée à fond, montre encore, dans les plus fins canaux lactifères, un résidu de lait très riche en matière grasse ; il est même probable que toute la graisse sécrétée n'est jamais soutirée, apporterait-on à la traite toute l'application voulue. Eckies et Shaw, dans un intéressant travail (1), acceptent l'idée de Kirchner, et ils ajoutent que, en général, plus une vache donne de lait, plus la différence entre le premier lait tiré et le dernier est grande, parce que, avancent-ils, chez une forte laitière la congestion en quelque sorte physiologique de la glande tend à obstruer les canaux d'évacuation du lait.

Le soubattage de la glande mammaire avant la mulsion. - Chez une petite laitière, dirai-je de mon côté, la différence s'atténue parce qu'il y a davantage de place pour loger le lait, et le soubattage de la glande pendant la mulsion tend à donner mécaniquement un mélange homogène asant même que le lait ne soit sorti de la glande. Sur ce point particulier, il y a lieu de citer une courte étude de Ragsdale, Brody et Turner :

A.-C. Ragsdale, S. Brody et Ch. W. Turner (2) se sont adressés à une vache Jerseyaise et ont fait des prélèvements successifs de 100 cc. sur un seul quartier, dans des circonstances différentes :

a) Immédiatement en arrivant à l'étable, venant de la pâture ;

b) Après un séjour de deux heures dans la tranquillité à l'étable;

c) Après un séjour de même durée, mais en ayant soin, avant la traite, de masser fortement la mamelle pendant quelques minutes, dans le but de mélanger le lait qu'elle contient. Le graphique joint fig. 28 nous donne tous les renseignements numériques utiles; il est extrêmement suggestif. Il nous montre combien le massage de la mamelle asant la traite tend à uniformiser le taux butyreux des divers prétèsements, notamment des premiers.

L'égalité des taux butyreux des divers prélèvements n'est évidemment pas assurée jusqu'à la fin, parce qu'on n'agite pas une mamelle comme une éprouvette dans laquelle la matière grasse serait en train de monter.

(1) Ch. Eckles et Roscoe-H. Shaw, Variations in the Composition of Milk from the Individuals Cows (Bull. 157. Bur. of Ann. Ind., Dept. of Agriculture, Washington, 1913).

(2) A.-C. Ragsdale, S. Brody et Ch.-W. Turner, the Variation in the percent of Fat in successive Portions of Cow's Milk (The J. of Dairy Science, t. IV, p. 448,1921$)$. 
Les auteurs prennent acte des chiffres donnés par leurs recherches et de ceux qu'ils ont recueillis en faisant des prélèvements dans une éprouvette pleine de lait à différentes hauteurs, soit au bout d'une heure et demie de repos du lait, soit au bout de trois heures pour donner la même cause aux résultats obtenus dans la mamelle, d'une
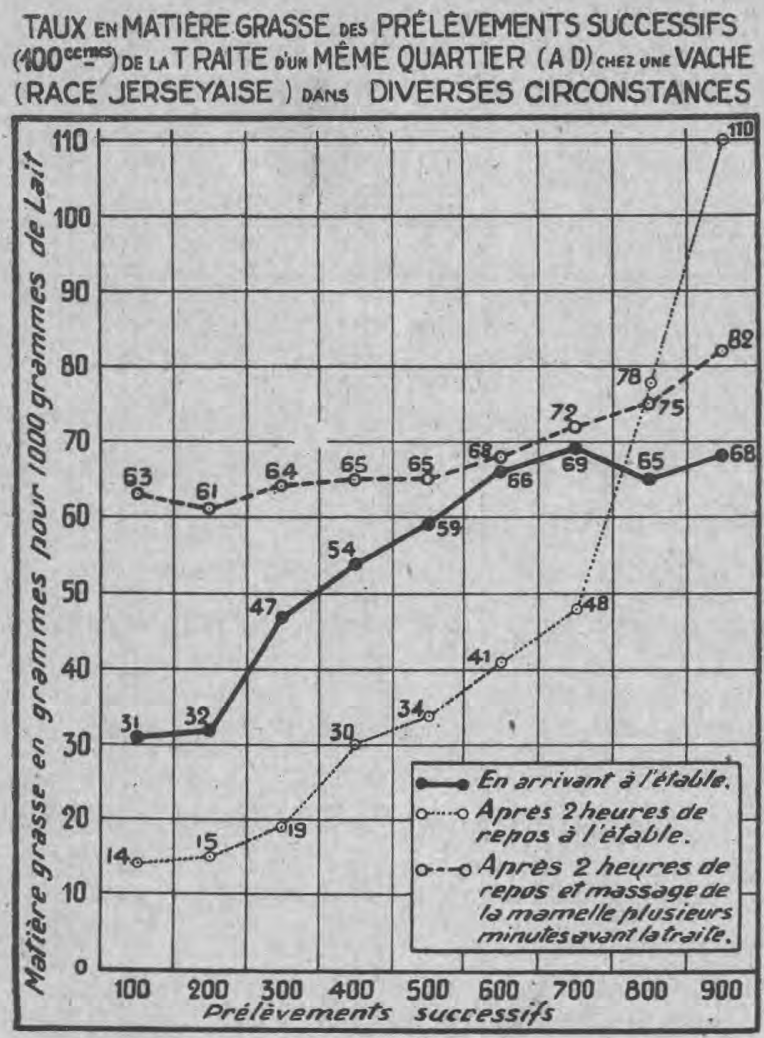

FIG. 28.

part, et dans l'éprouvette, d'autre part; c'est revenir à la théorie de SANSon qui admet que c'est la différence de densité entre la matière grasse et le lait écrémé qui intervient dans la mamelle, chèz l'animal vivant, aussi bien que dans le récipient en verre.

Je me permettrai d'être d'un avis tout à fait différent, en faisant remarquer que les situations sont loin d'être les mêmes. Nous avons dans la mamelle, en considérant le sens centrifuge, des canaux dont le diamètre s'exagère au fur et à mesure que l'on s'écarte des acini mammaires; l'adhérence des globules contre les parois des canaux les plus éloignés de la porte de sortie du lait est évidemment beau- 
coup plus forte, en raison de l'extrême petitesse du diamètre de ces derniers.

J'ajouterai que la théorie, qui suppose que la cause principale des variations du taux butyreux des divers prélèvements de la traite tient à l'adhérence des globules gras contre les petites parois lactifères, trouve un appui dans deux faits, l'un qui est relatif à l'accroissement du diamètre des globules gras, à mesure que la traite s'avance, - nous en parlerons tout à l'heure -, l'autre qui nous. montre qu'il en est pour les leucocytes comme pour les globules gras; le taux leucoeytaire des divers prélèvements s'élève au fur et à mesure que l'on approche de la fin de la traite, parce que la gymnastique à laquelle est soumise la glande pendant cette opération et la continuité du flot lacté dans le sens centrifuge finissent par décoller les leucocytes.

Le graphique 28 montre que plus longue est la durée du repos auquel sont soumis les animaux, quand ils sont rentrés à l'étable, avant la traite, plus grande est la différence dans le taux butyreux du premier et du dernier prélèvement.

Il n'est done point besoin d'avoir recours ici à la différence de densité entre la matière grasse et le lait écrémé ; faisons simplement. remarquer, ce qui est aussi très plausible, que le repos ne peut que faciliter l'accolement des globules gras contre les parois des canalicules les plus fins de la glande.

Dans le travail cité plus haut, Eckles et Shaw montrent que les gros globules sont plus facilement retenus que les fins; aussi sont-ils en plus forte proportion dans le dernier lait; c'est dans le lait du début que l'on note les plus grandes variations dans les dimensions des globules, les plus petits s'y trouvant surtout. La fig. 29 reproduit graphiquement le cours de l'enrichissement du lait du commencement à la fin de la traite, chez des vaches de races différentes, en même temps qu'il nous donne les dimensions moyennes relatives des globules gras dans les eent premiers et les cent derniers centimètres cubes recueillis, comparées à celles des globules de la traite entière.

Nécesisité de faire la traite à fond. - Les variations, si grandes parfois, entre le premier lait recueilli et le dernier, montrent combien il importe d'opérer la traite à fond, tant au point de vue économique, afin de recueillir le dernier lait, le plus riche en matière grasse, qu'au point de vue pénal.

Le lait, pour répondre à la définition qu'en donnent les règlements officiels dans tous les pays - et c'est le cas du décret du 25 mars 1924, pour la France, - doit provenir d'une traite complète. Les 
Le lait du début et celui de la fin de la traite comparés à celui de la traite entière.

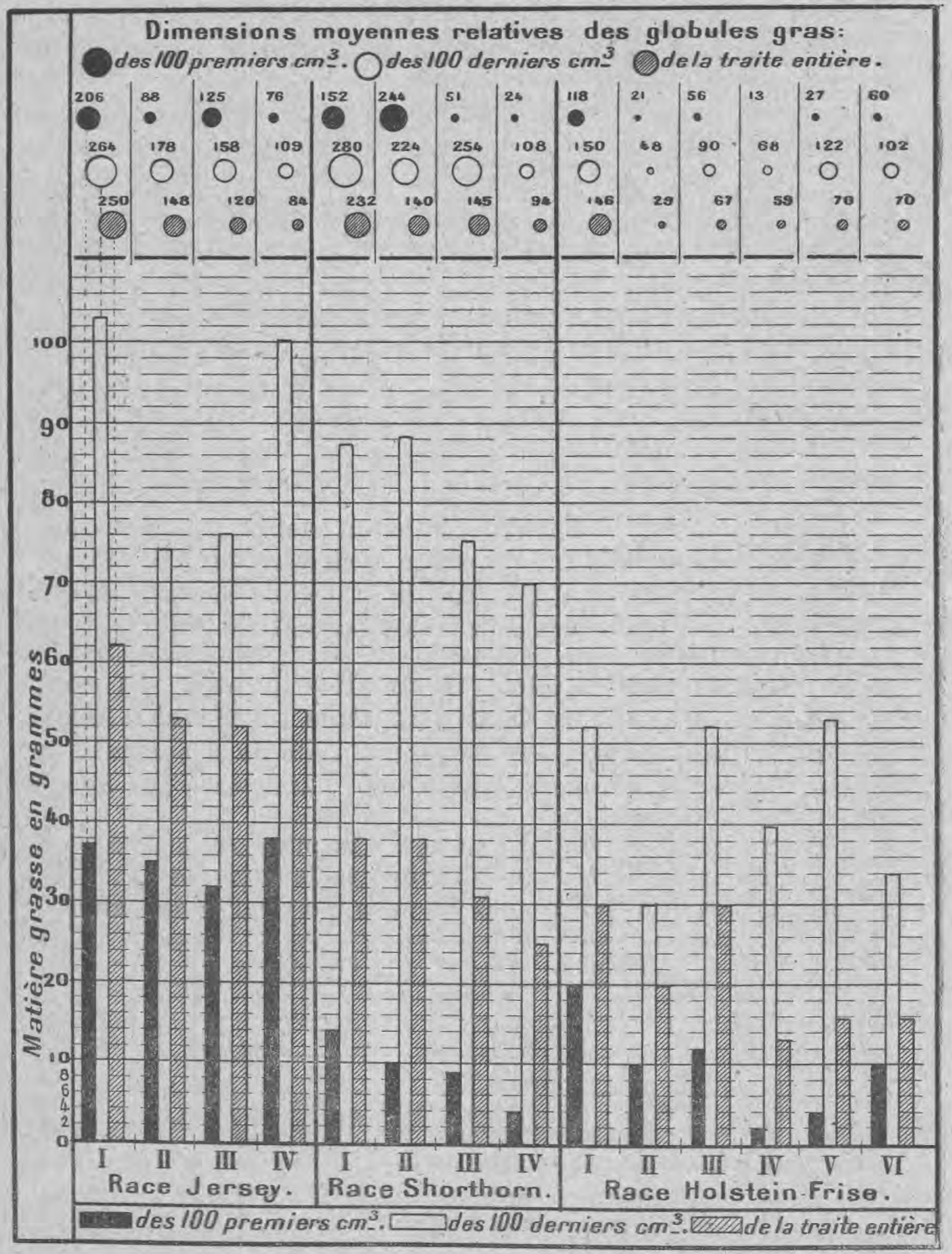

FIG. 29.

derniers documents ci-dessus nous montrent péremptoirement que toute traite incomplète peut fournir, et fournit dans la grande majorité des cas, un lait ayant les caractères d'un lait écrémé. Souvent le frau- 
deur, pour expliquer la faible richesse en matière grasse du lait saisi, avance que la traite a été mal effectuée, incomplètement poussée à fond, par un vacher inexpérimenté ou pressé de finir son travail, et que lui ne saurait, en bonne justice, en être rendu responsable.

Ce raisonnement, évidemment, a sa valeur, - et remarquons en passant qu'il ne peut s'appliquer qu'à un lait individuel, - mais il ne saurait être retenu, d'abord parce que le producteur doit endosser la responsabilité de son personnel, et ensuite j'ajouterai que le fait pour lui de reconnaître que le lait de la fin de la traite est le plus riche en matière grasse ne saurait disposer le juge en sa faveur puisqu'il n'ignore rien du geste qui lui est reproché.

Faire une traite incomplète, ou du moins ne livrer que le produit d'une traite incomplète, e'est, comme le dit 'M. Boproux, faire de l' "écrémage au ventre », opération qui est illicite.

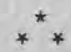

L'unicité de la fonction mammaire étant établie pour l'ensemble des espèces mammifères, l'enrichissement progressif du lait en matière grasse, du commencement à la fin de la traite, constaté chez la vache, s'observera également chez les autres espèces.

L'enrichissement du lait en matière grasse du commencement à la fin de la traite chez la chèvre, de la tétée chez la femme. - Voici une suite de documents empruntés encore à l'excellent travail de CaIlloux. La fig. 30

Taux, en grammes, par litre, de la matière grasse dans les trois fractionnements de la traite ( effectuée chez une chèvre, sur chaque quartier séparément.

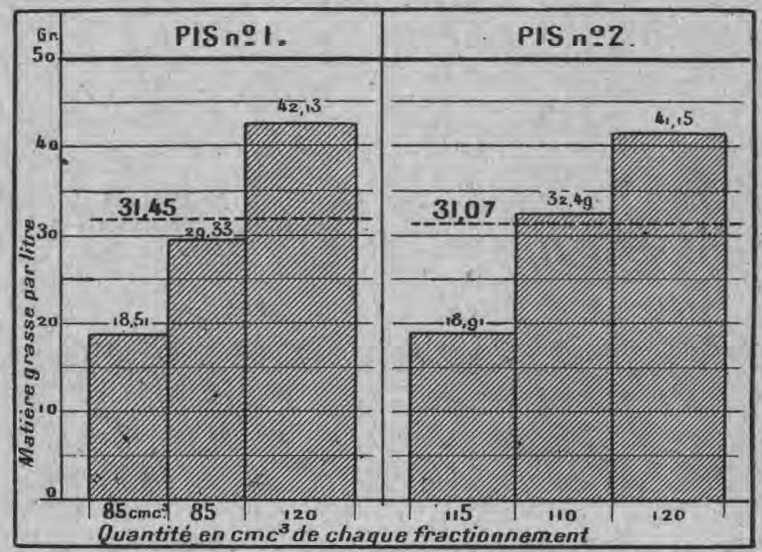

FIG. 3o. 
est relative à la chèvre. Dans la fig. 31 , on trouvera les variations de la richesse butyreuse du lait sur les divers prélèvements

Variations de la richesse butyreuse du lait pendant la tétée. Taux, en grammes, par litre, de la matière grasse dans le lait des divers fractionnements de la tétée chez une femme peu beurrière (sein droit).

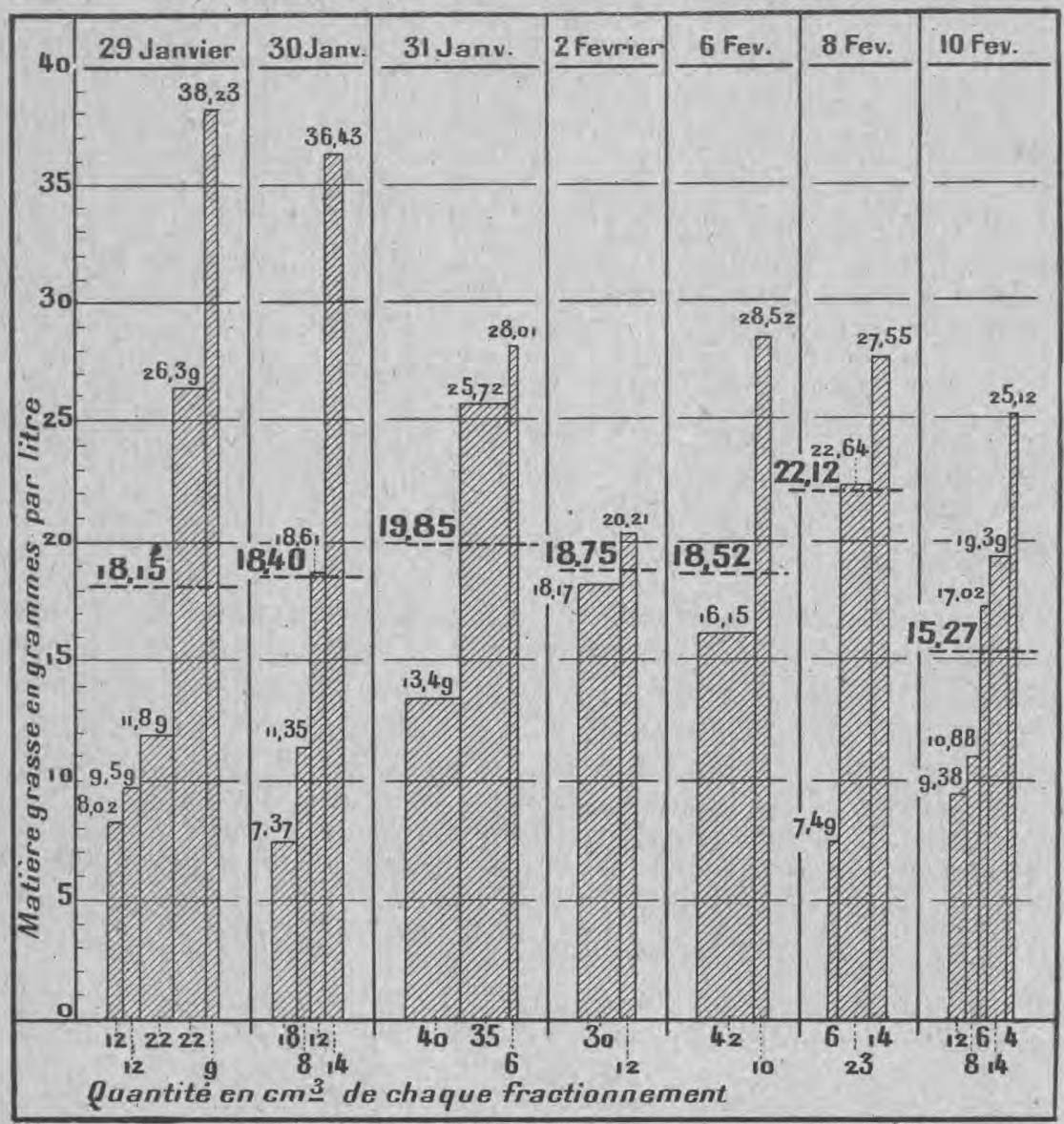

FIG. 31.

d'une tétée faite à fond chez une femme, pendant plusieurs jours, se suivant ou rapprochés, au sein droit. Sur les fig. 32 et 33 , ce sont les variations journalières de la tétée fractionnée du sein droit (fig. 32), du sein gauche (fig. 33), pendant vingt-cinq jours consécutifs.

Notons, en passant, ce fait important que la fig. 31 nous révèle : 
il s'agit d'une femme dont le lait est peu butyreux, ainsi qu'en font foi les 7 moyennes butyreuses du lait total en 12 jours, moyennes qui sont données en caractères plus gros; au contraire, l'allure des courbes des fig. 32 et 33 nous indique qu'il s'agit d'une femme dont le lait est nettement plus riche en matière grasse.

La fig. 34, qui résume des observations du travail de PlauCHU et Rendu, et dans lesquelles il n'est tenu compte que des laits du commencement et de la fin de la tétée, et non pas de l'ensemble des prélèvements, comme dans les observations de Carluoux, présente, malgré cette lacune, un grand intérêt. Il apparaît bien que les deux premières nourrices, Les... et Mont... ont un lait qui est moins riche que celui de la troisième nourrice, Jacq... Les trois nourrices en question ont été examinées plusieurs fois, quatre pour la première et la seconde, cinq pour la dernière. Dans l'ensemble, les analyses de chacune sont concordantes, aussi la conclusion que nous venons d'en tirer est-elle valable. Toutéfois, il importe de faire remarquer, afin de passer à còté de l'erreur dans laquelle certains pourraient tomber, que, pour apprécier la richesse moyenne d'un lait - et, bien entendu, ce n'est que d'un lait de traite complète qu'il peut s'agir, chez la femme ou une autre espèce, - il ne faudrait pas se contenter de faire la moyenne du lait du début et du lait de la fin de la traite ; on risquerait de se tromper étrangement. Une pareille moyenne ne peut être équitablement établie que si l'on tient compte des quantités séerétées aux divers prélèvements sucessifs, en affectant à chacun son taux butyreux propre ainsi qu'il a été fait pour les fig. $25,26,30$ et 31 . Or, nous allons voir que cette erreur, malgré toutes les précautions prises, est faite, en général, pour le lait de femme, dont elle charge ainsi les chiffres de l'analyse d'une inconnue difficile à apprécier.

\section{***}

L'échantillon moyen chez la femme. - Ainsi que nous l'avons fait remarquer plus haut, s'il est toujours facile d'avoir un échantillon moyen sincère avec la vache, la chèvre et la brebis, la chose est fort difficile, pour ne pas dire, dans l'immense majorité des cas, impossible, lorsqu'il s'agit de la femme. Aussi, le prélèvement des échantillons chez la nourrice a-t-il de tout temps préoccupé les chercheurs.

Dans sa thèse (Paris, 1859), Covderkau dit : a Il faudrait, pour avoir une moyenne vraie de sa composition (lait de femme), prélever à chaque tétée une petite quantité de lait au commencement et à la fin du repas de l'enfant. " Je viens de faire remarquer ce qu'une pareille technique peut comporter d'erreur, en m'appuyant sur les documents qui précèdent. 
Variations jcurnalières de la traite fractionnèe du même sein (droit) chèz une jeune femme quadripare de 32 ans, pendant 25 jours consécutifs.

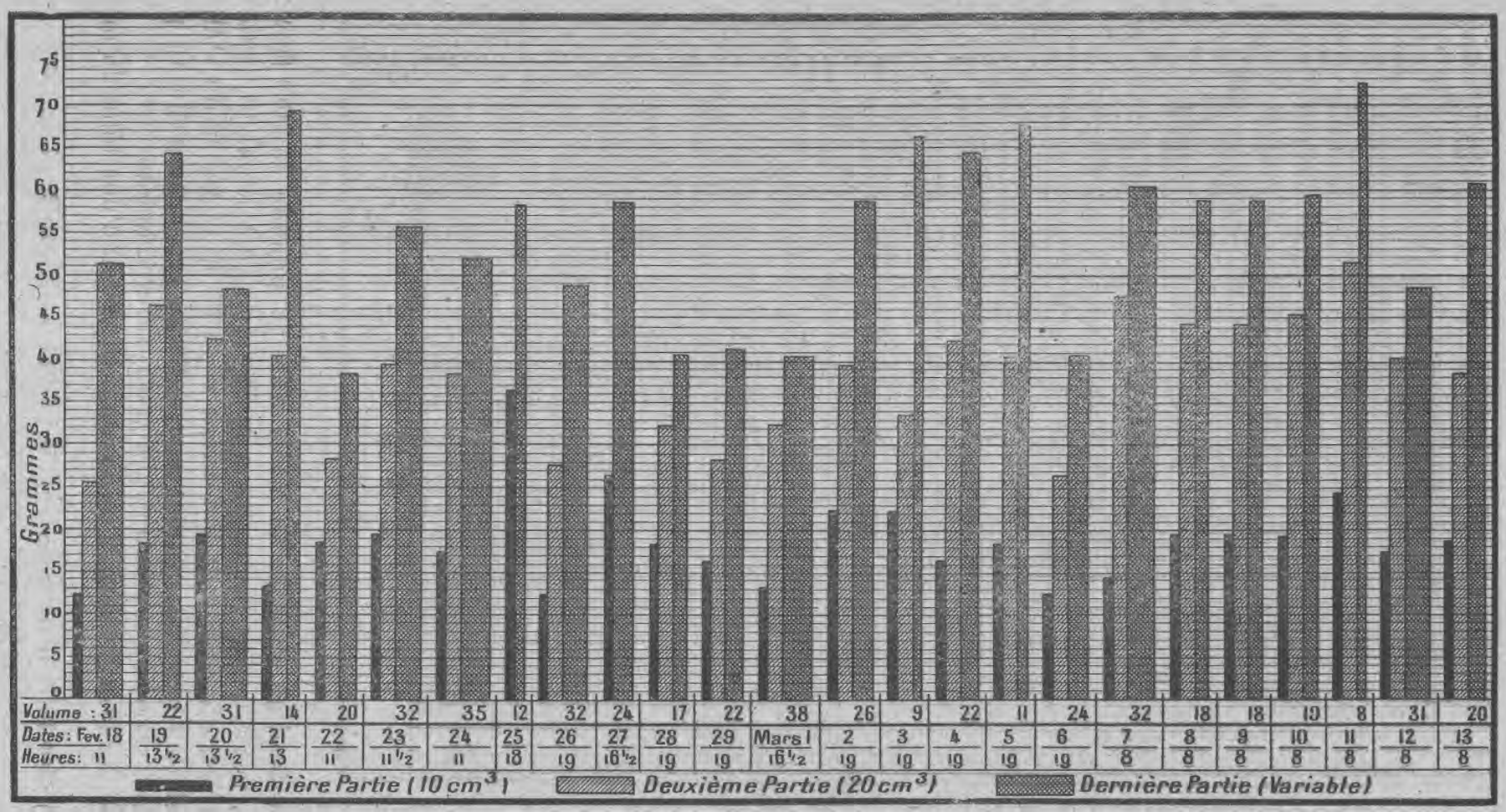




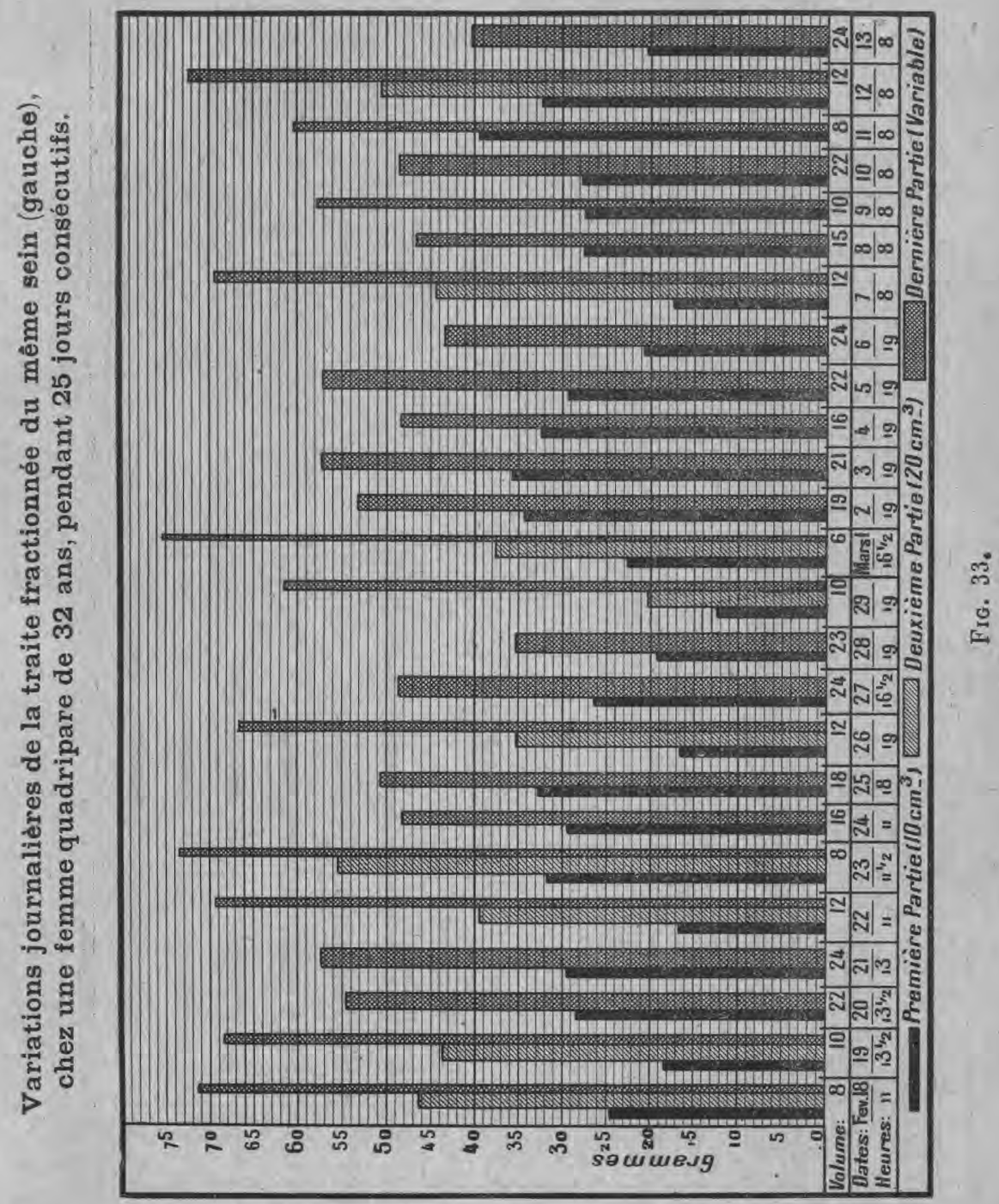

Je ferai un reproche du même ordre au procédé de $M^{\text {me }} B$ RÈs"(1) qui, pour obtenir l'échantillon moyen destiné à l'analyse, prélevait une petite quantité de lait avant et après la tétée, et du mélange qu'elle en obtenait, prenait une quantité proportionnelle au poids de lait absorbé par l'enfant à la tétée correspondante.

Ch. Miches prélève $20 \mathrm{cc}$. au commencement d'une tétée du matin,

(1) Mme Brès, De la mamelle et de l'allaitement, Thèse de Paris, 1875. 
20 ec. au milieu d'une tétée de midi et 20 cc. à la fin d'une tétée du soir (Ch. Miches, Obstétrique, mars 1906) et l'analyse était faite sur le mélange de ces trois prélèvements:

Ch. Marchand (De la composition anormale que peuvent présenter certains laits de fenme, Ass. Franc. pour l'Apanc. des Sciences, le

Taux, en grammes, par litre, de la matière grasse, du début et de la fin de la têtée, chez plusieurs nourrices.

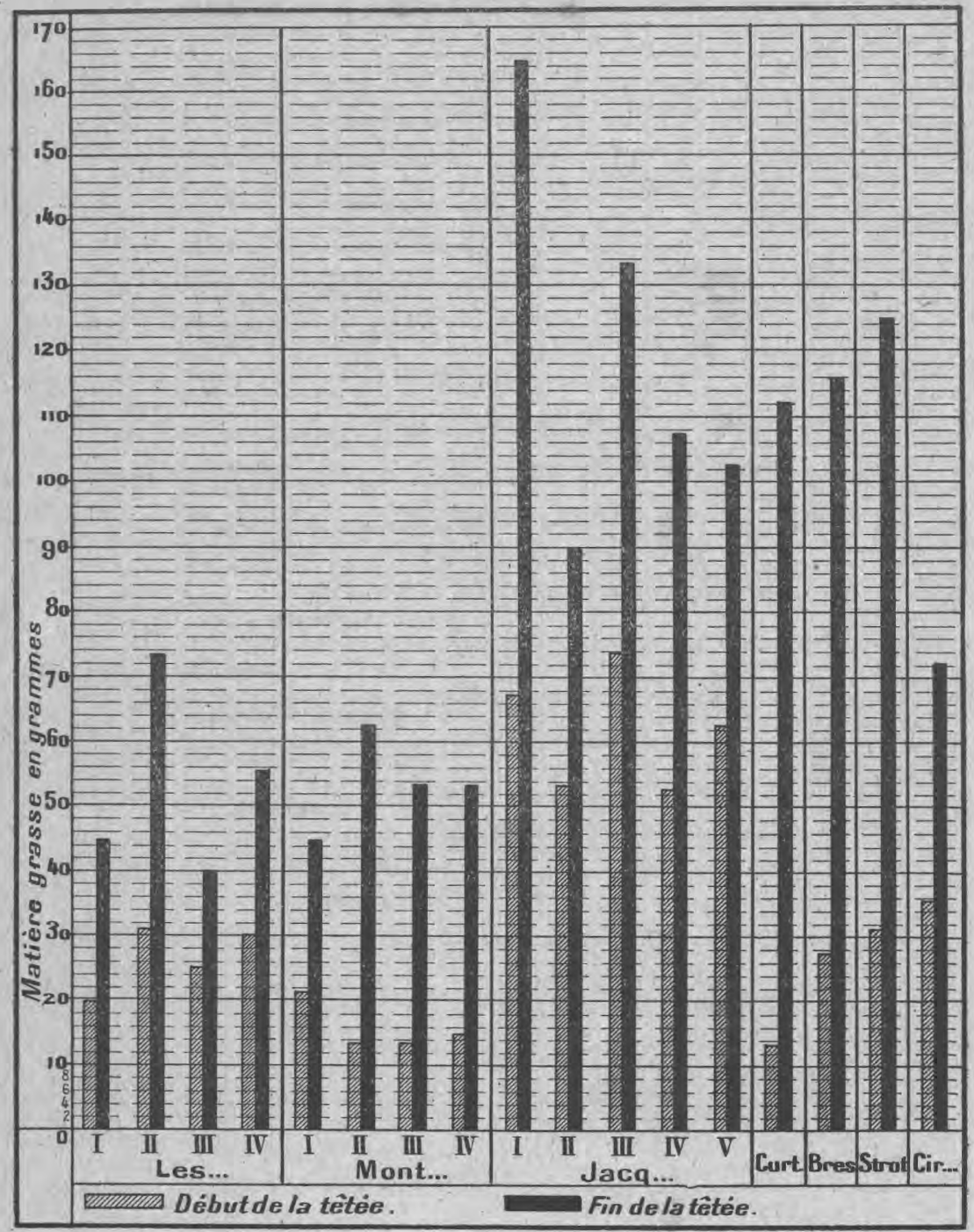

FIG, 34. 
Havre, 1877 , p. 877) croit nécessaire que le temps écoulé depuis la dernière traite n'excède pas deux ou trois heures, et il prélève l'échantillon après que l'enfant a tété quelques instants.

Gurraud (1), prenant exemple sur H. Férx, suit une technique un peu analogue à celle de Marchand. It prélève l'échantillon le matin, une heure et demie à deux heures après que l'enfant a tété ; met l'enfant au sein pendant quelques instants afin de provoquer la sortie du lait, pour que la récolte s'effectue mieux.

Pratehu et Rendu, dans leurs recherehes qui ont porté sur 3.450 échantillons de matière grasse, ont toujours pris le lait du début : "On sait toujours, disent-ils, quand est le début d'une tétée, tandis qu'on ignore quand c'est le milieu ou la fin. " Cette réflexion est trop facile pour ne pas être juste, mais il n'en est pas moins vrai que la technique de ces auteurs est entachée d'une faute, puisque les analyses ne portent toujours que sur un lait, dont le moins qu'on puisse dire est que sa composition ne saurait être comparée à celle du lait moyen.

Malgxé cette réserve, nous avons vu que la courbe de la richesse butyreuse du lait chez une même femme subit des oseillations considérables d'un jour à l'autre, et rentre ainsi dans le cadre des courbes des autres femelles laitières.

Denis et ТАLвот (loc. cit.) recuellent 30 gr. de lait avant que l'enfant tête, puis 30 autres grammes après que l'enfant a tété pendant vingt minutes; c'est sur le mélange de ces deux prises que l'analyse est faite. (Voir le tableau de la page 379.)

Ce que j'ai dit tout à l'heure de l'importance qu'il y a à soubattre la mamelle avant de tirer le lait, afin de produire une agitation mécanique susceptible de décoller les globules gras fixés sur les parois des petits canaux lactifères, porte à penser que le malaxage du sein, effectué pendant plusieurs minutes avant la traite, nous permettrait sans doute d'avoir un premier prélèvement dont la composition serait plus voisine, au point de vue de la matière grasse, de celle du Tait moyen, que celle du premier lait tiré, sans malaxage préliminaire. Il m'apparait possible que l'on pourrait, guidé par ee qui vient d'être dit, effectuer des expériences analogues chez la femme et les femelles laitières; jusqu'ici, on n'a que celle de Ragsdale, Brody et Túner sur la vache.

Au surplus, quand on y réfléchit, on peut penser que l'argument avancé par Plauchu et Rendu, et avec lequel ils justifient leur manière de procéder, n'est pas aussi valable qu'il semble l'ètre à

(1) Guiraud, le Lail de femme, Thèse de Bordeaux, 1897. 
Analyses de laits du commencement et de la fin d'une seule tétée.

\begin{tabular}{|c|c|c|c|c|c|c|c|}
\hline \multirow{2}{*}{\multicolumn{4}{|c|}{ AGE DE L'ENFANT }} & \multirow{2}{*}{$\begin{array}{c}\text { AGE } \\
\text { DE LA } \\
\text { MÉRE }\end{array}$} & \multirow{2}{*}{$\begin{array}{c}\text { NOMBRE } \\
\text { DE } \\
\text { GROSSESSES }\end{array}$} & \multicolumn{2}{|c|}{$\begin{array}{l}\text { MATIÈRE GRASSE } \\
\text { pour r.ouo gr. de lait }\end{array}$} \\
\hline & & & & & & Premier lait & Dernier Iait \\
\hline 3 & semaines & . . & & 18 ans & 2 & 53 & 64 \\
\hline 3 & - & .. & & $18-$ & 2 & 89 & 109 \\
\hline 2 & - & $\therefore$ & : & $18-$ & 1 & 15 & 52 \\
\hline 9 & - & . & & $38-$ & 1 & 50 & 134 \\
\hline 9 & - & & & ) & " & 5,8 & 74 \\
\hline 17 & jours. . & . . & & $18-$ & 1 & 67 & 71 \\
\hline 4 & semaines & $\therefore$ & & $16-$ & 2 & 71 & 49 \\
\hline 4 & - & $\therefore$ & & $16-$ & 2 & 74 & 74 \\
\hline 4 & 1 & . . & & $18-$ & 1 & 40 & 80 \\
\hline 3 & - & . & & $18-$ & 1 & 64 & 160 \\
\hline 27 & - &.$\therefore$ & & " & ) & 24 & 80 \\
\hline 4 & - & . . & . & $18-$ & 2 & 74 & 110 \\
\hline 4 & - & & & n & ) & 56 & 52 \\
\hline 4 & - & 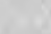 & & " & ” & 24 & 38 \\
\hline 26 & semaines & .. & . & $26-$ & 2 & 25 & 44 \\
\hline 18 & jours. . & . . & . . & $20-$ & 2 & 16 & 14 \\
\hline 18 & semaines & . . & & $28-$ & 1 & 20,6 & 12,2 \\
\hline 5 & - & . & . & $18-$ & 2 & 56 & 71 \\
\hline 5 & - & . & . & $22-$ & 1 & 56 & 91 \\
\hline 7 & - & . & & $21-$ & 1 & 32 & 66 \\
\hline 6 & - & . & . & $16-$ & 1 & 45,2 & 80 \\
\hline 9 & - & . & . & $22-$ & 1 & 64,8 & 133 \\
\hline 9 & - & & & ") & » & 24,8 & 54 \\
\hline 9 & - & & & ” & ” & 15 & 29 \\
\hline 40 & semaines & $\therefore$ & . & ” & 3 & 35 & 47,6 \\
\hline 22 & - & . . & - . & ” & 2 & 32 & 27 \\
\hline 22 & - & 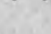 & & n & \% & 42 & 72,4 \\
\hline 40 & semaines & . & . & ” & 6 & 6,6 & 100 \\
\hline 43 & - & . & . & $n$ & ) & 42 & 36 \\
\hline 49 & - & . & . & 川 & » & 15 & 80 \\
\hline 26 & jours. . & .. & $\therefore$ & $25-$ & 2 & 29 & 51 \\
\hline 25 & $-\cdots$ & . & . & $23-$ & 1 & 42 & 41 \\
\hline 39 & semaines & & . & n. & ) & 55 & 131 \\
\hline 39 & - & 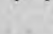 & & \# & 》) & 29 & 55 \\
\hline 10 & semaines & & & $29-$ & 3 & 30 & 45 \\
\hline 3 & - & . & & $24-$ & 3 & 16,6 & 32 \\
\hline 3 & - & . . & & n & 1 & 26,4 & 36,8 \\
\hline 2 & - & . & & $26-$ & 3 & 1 & 33 \\
\hline 25 & jours.. & . & & $20-$ & 1 & 16. & 20,2 \\
\hline 4 & semaines & . & & $19-$ & 1 & 26 & 36 \\
\hline 26 & _ & . & & $35-$ & 3 & 20 & 28 \\
\hline 2 & - - & . & . & $24=$ & 1 & 17 & 40 \\
\hline 4 & - & & & $21-$ & 3 & 19 & 24 \\
\hline 4 & - & & & (1) & $n$ & 84 & 84 \\
\hline 4 & - & & & ” & » & 17. & 46 \\
\hline 4 & - & & & » & $"$ & 13,9 & 36,8 \\
\hline
\end{tabular}

(Tableau emprunté au travail de Denis et TALbot, v. p. 378). 
première vue. Il aurait quelque valeur si l'importance de la tétée entière était, à peu de chose près, invariable; mais il n'en est pas ainsi, et les premiers $10 \mathrm{cc}$. d'une tétée dont le total doit atteindre $50 \mathrm{ce}$, ne sont nullement comparables aux mêmes 10 premiers centimètres cubes de la tétée totale du même sein, un autre jour, si celle-ci devait atteindre 70,80 ou même 100 cc. ; aussi, sans paradoxe aucun, on peut dire que l'on ne sait pas plus quand e'est le commencement de la tétée que lorsque c'est la fin. Dans un cas, comme dans l'autre, la question de relativité des valeurs des divers prélèvements retentit grandement sur l'importance que l'on doit attacher à l'échantillon prélevé.

Carlloux, dont les recherches ont porté sur des tétées totales, ce qui lui a permis de constater combien variable était la teneur en matière grasse aux différents prélèvements de la traite, s'est servi du tire-lait de Budin relié à un tube à vide, et, poưr diviser la traite, le flacon dans lequel on recueille le lait comporte un dispositif semblable à celui qu'on utilise dans les laboratoires pour les distillations fractionnées dans le vide.

$\mathrm{Si}$, chez les femelles laitières orientées en vue du fonctionnement intensif de leur glande mammaire, il est interdit de recourir à un simple échantillon, serait-il même un échantillon moyen d'une journée entière pour apprécier la qualité butyreuse de l'animal, une pareille interdiction s'impose davantage encore chez la femme, en raison de l'absence presque régulière chez celle-ci de l'échantillon moyen.

On doit toujours faire preuve d'une grande circonspection lorsque l'on veut donner au choix de la nourrice une base analytique chimique. Il faut multiplier les prélèvements, comme l'ont fait Plauchu et ReNdu pour les nourrices Lès..., Mont... et Jacq... En opérant ainsi, on se garde dans une certaine mesure des erreurs d'interprétation dans lesquelles on pourrait tomber avec l'analyse d'un seul prélèvement et l'on tend à se rapprocher de la vérité.

Il faut toujours avoir à l'esprit les chiffres surprenants que l'on obtient avec la vache, et qui nous interdisent d'apprécier celle-ci sur la base d'un seul examen chimique, et se dire que chez la femme il en est plus qu'ainsi, puisqu'aux difficultés produites par la grande variabilité du taux butyreux, s'ajoutent celles du prélèvement.

Je dirai encore, au sujet de la nourrice, que nous laissons de côté, n'ayant en vue ici que la matière grasse, la partie non grasse du lait; or, celle-ci a tout autant d'intérêt que celle-là; les erreurs de jugement dans le choix d'une nourrice, qui dérivent de l'interprétation incomplète et tendancieuse d'un seul lait qui peut être mal prélevé, peuvent être donc grandes. 
La variabilité du taux butyreux du lait en face du facteur alimentation. - La lecture du grand nombre de documents que nous venons de donner n'aura pas été sans faire naître dans l'esprit du lecteur quelques réflexions, mais il en est une qui n'aura pas manqué de surgir presque tout de suite : puisque le taux de la matière grasse parie dans des limites nussi considérables, alors que l'animal, en bonne santé, pit dans des conditions toujours semblables, et reçoit une alimentation constamment la même en qualité et en quantité, il semble donc très difficile d'établir des relations directes, étroites, de cause à effet, entre l'alimentation et la matière grasse du lait, au point de vue quantitatif.

Dans la première partie de cette étude, nous avons, en nous appuyant sur des considérations physiologiques de bonne qualité, croyons-nous, et sur des expériences auxquelles on peut accorder tout crédit, développé les raisons pour lesquelles l'aliment ne pouvait avoir qu'un retentissement faible, très lointain, sur la quantité de la matière grasse sécrétée par la mamelle. Nous avons vu dans quelle mesure l'aliment agissait qualitatipement, mais nous avons moins insisté pour voir dans quelles mesures il lui est possible d'agir quantitatipement, parce que nous attendions d'avoir fait étalage de tous les chiffres qui précèdent, pour discuter avec intérêt de ce point particulier.

Tandis que la cause ou la prétendue cause : l'alimentation, à laquelle beaucoup d'esprits, plutôt mal que bien éclairés, donnent tant d'importance reste constante, et il suffit pour cela d'assurer à l'animal des repas identiques, qualitativement et quantitativement, pendant une période assez longue de temps, que voyons-nous? Le taux en matière grasse de son lait osciller considérablement, d'une traite à l'autre, d'un jour à l'autre, et si nous serrons davantage encore le problème, pour la mème traite, d'un quartier à l'autre. Où placer ici, en présence de telles variables, l'influence de l'aliment?

Les problèmes les plus difficiles de la physiologie animale sont, sans nul doute, ceux qui touchent au métabolisme. Le champ très vaste des recherches qui portent, d'une part, sur la dislocation des molécules alimentaires et, d'autre part, sur la reconstruction des molécules de l'être vivant aux dépens des morceaux des premières, est extrêmement difficile, pour ne pas dire impossible à aborder directement.

C'est par des moyens indirects que l'on peut parfois résoudre les problèmes posés; aussi les réponses à la question de savoir quelle est l'origine des principes du lait, et notamment celle de la matière grasse, ne peuvent être que partielles. 
Nous avons vu en commençant que la croyance la plus simpliste, ou du moins la plus ancienne, était que la graisse du corps, tout comme celle qui est éliminée par le lait, provenait directement de la graisse des aliments, par un simple transfert en quelque sorte de celle-ci aux tissus de l'organisme ou à la mamelle.

Une telle hypothèse n'est justifiée que dans une certaine mesure ; elle est invraisemblable prise dans son acception totale, et cela éclate tout de suite aux yeux quand on constate qu'avec des aliments identiques la femelle laitière fera des graisses différentes : suif, graisse des os, beurre. Rappelons, pour montrer la complexité du problème, qu'il est hors de contestation que la graisse peut se former, et e'est indiscutable chez les herbivores, aux dépens des hydrates de carbone.

La physiologie animale nous dit qu'à l'heure actuelle on sait relativement peu de choses encore sur le métabolisme des matières grasses; on en sait davantage sur celui des hydrates de carbone, et aussi sur celui des matières protéiques, depuis que nos connaissances sur la constitution de ces dernières se sont très élargies.

Il serait évidemment guère scientifique de prendre acte du peu que nous savons sur le métabolisme des matières grasses pour nier une influence alimentaire quelconque, mais quelle est la grandeur de cette influence, dans quel sens agit-elle? Nous n'avons jamais que des ébauches de réponse à ces questions.

Chez la vache, chez la chèvre, chez la brebis, la graisse n'est pas seule à intervenir; les hydrates de earbone, nous le savons, vont faire des graisses de réserve qui, à leur tour, serviront à faire de la graisse du lait; il y a donc là une transformation intermédiaire qui complique une interprétation à laquelle des esprits superficiels ne trouvent évidemment aucune difficulté.

Si j'insiste sur ces points - trop mème dira-t-on - c'est parce que les fraudeurs font jouer l'inflyence de l'alimentation plus que de raison. En général, quand survient un changement de régime, il n'y a pas que l'alimentation qui varie; de nombreux circumfusa tenant à l'atmosphère, la température, l'humidité, le climat, etc., interviennent. Un changement de régime ce n'est pas seulement une modification dans l'apparence extérieure de la ration des animaux; il y a bien des inconnues qui nous échappent. Quand on substitue le régime vert au régime sec, la nutrition reçoit un coup de fouet. Ce que nous savons aujourd'hui de l'influence de eertaines vitamines dans le métabolisme de la chaux, par exemple, ne nous permet-il pas de penser que derrière l'influence des grosses molécules alimentaires se rattachant à un type connu : hydrates de carbone, graisses et protéines, il en est d'autres relevant de principes sur lesquels qualitativement et quantitativement nos connaissances sont faibles? 
Dans un travail plein de suggestions intéressantes (1), Maynard et Mrers, étudiant les facteurs qui jouent dans les expériences relatives à l'influence de l'alimentation sur la riehesse du lait en matière grasse, distinguent entre les facteurs mesurables et les facteurs non mesurables. Dans les premiers, ils reconnaissent l'espèce et la quantité de l'aliment, la méthode qui préside à l'alimentation : heures des repas, etc. Il est d'autres de ces facteurs qui, bien que ne pouvant pas être évalués exactement, rentrent cependant dans le même groupe, telles que les conditions de vie : à l'intérieur ou au dehors, la température, etc.

Dans les facteurs non mesurables, il faut placer toutes les particularités physiologiques : qualité de la nutrition, valeur de la production, qui toutes se rattachent à I'individualité. Celle-ci ne peut pas être contrôlée; elle se constate, mais du moins en peut-on mesurer les effets.

Maynard et Myers font remarquer, avec juste raison, combien l'interprétation des résultats se heurte à beaucoup plus de difficultés quand il s'agit de lait que lorsqu'il s'agit de viande; a fortiori, dirai-je, quand on envisage un seul constituant du lait, celui qui est le plus variable : la matière grasse.

Quand on vise à faire de la viande, e'est l'augmentation du poids de l'animal qui est avant tout recherchée, et toute nourriture, consommée en sus de celle qui est nécessaire pour assurer l'entretien, est profitable. Dans l'alimentation de la femelle laitière, seul, ce qui intéresse, c'est la production de la glande mammaire; aussi, l'augmentation du poids de ladite femelle au cours d'expériences d'alimentation est-elle indésirable ; tout engraissement ne peut que tendre à nuire à l'activité de la glande mammaixe, parce qu'il dérive ailleurs une partie plus ou moins importante de ce qui est.réclamé par eette dernière.

La production du lait est sujette, beaucoup plus que celle de la viande, à de plus larges variations et à l'influence de facteurs qui viennent rapidement la troubler.

Armsby, dans son Tivre (the Nutrition of Farm Animals, 1905, p. 500), dit : "L'alimentation de la femelle laitière est, dans une certaine mesure, un facteur secondaire en laiterie; le succès dans la production laitière dépend : $1^{\circ}$ de l'individualité de l'animal et de toutes les circonstances qui permettent à celle-ci de jouer librement. L'alimentation ne peut pas stimuler grandement la production, bien qu'elle puisse cependant la diminuer s'il y a manque de

(1) L.-A. Maynard et W. Mrens, the Refinement of feeding experiments for milk production by the applieation of statistiscal methods. (Bull.397 de Cornell University, avril 1918). 
substances nutritives. Les rations impropres, suffisantes quantitativement, mais insuffisantes qualitativement, peuvent nuire à la production du lait et favoriser celle de la viande... "

La sécrétion lactée est tout d'abord une fonction périodique, et son allure présente des irrégularités, difficilement explicables, soit qu'on la considère chez différents animaux ou chez le même animal au cours de lactations différentes.

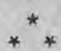

\section{L'appréciation d'une femelle laitière au point de vue butyrogène en face des oscillations du taux en matière} grasse de son lait. - A première vue, de l'irrégularité de la courbe du taux butyreux du lait chez une femelle laitière, il semble qu'on puisse en déduire la grande difficulté à apprécier celle-ci au point de vue butyrogène. Toutefois, si l'on se reporte aux documents de cette étude, on constate, et le fait est assez net lorsque l'observation englobe un grand nombre de jours, qu'il est possible, derrière cette irrégularité qui comporte des oscillations très grandes et désordonnées, de juger si l'animal est un bon ou un médiocre producteur de matière grasse par son lait. Un pareil jugement doit s'appuyer sur des chiffres indiscutables, lesquels seront fournis par la mesure de tout le lait produit et la détermination de sa richesse en matière grasse.

Dans l'importante étude que le Lait a consacrée au Contrôle laitier (1), nous trouvons un grand nombre de ces chiffres qui sont, à n'en pas douter, éminemment suggestifs. Leur importance est liée étroitement à quelques données physiologiques qui ont été mises en valeur par les recherches de FLEISCHMANn et de ses élèves.

A. Mallèvre, dans une conférence remarquable (2), relève toute l'importance de ces dernières. Il est bien vrai que, empiriquement, on connaissait déjà très bien l'influence de l'individualité sur la richesse du lait, mais pour l'utiliser comme il convenait, " la faire servir, dit MALLÈvre, aux progrès de l'élevage et de la production laitière, on doit pouvoir l'apprécier, la mesurer et, conséquemment, la chiffrer. ")

Pour éliminer les influences soupçonnées, mais si difficiles à préciser, qui font varier si bizarrement la richesse du lait en matière grasse, il

(1) Le Lait no 7-8 de 1922 uniquement consacré au contrôle laitier, brochure de 180 pages.

(2) A. Mallìvre, Les variations de la richesse butyreuse du lait et les influences dont elles dépendent. Conséquences relatives à la production du lait et à la recherche de l'écrémage frauduleux (Société Centrale d'agriculture de la Seine-Inférieure. Bull. Trimestriel, octobre-novembre-décembre 1910).1 
faut, de toute nécessité, établir la teneur moyenne, butyreuse de l'animal pendant une lactation entière. Le moyen le plus certain, e'est celui auquel ont eu recours Fleischmann et son élève Hittcher ; ils ont pesé chaque jour le lait de chaque traite des vaches en expérience, et chaque fois ont dosé la matière grasse. Ils ont donc pu, en toute sûreté, avoir la richesse moyenne, et sont arrivés à cette remarquable conclusion : e'est qu'une vache donnée sécrète un lait dont la teneur moyenne butyreuse est à peu près insariable pendant sa carrière, à la condition, bien entendu, que la santé de l'animal ne subisse aucune atteinte, et que la nourriture soit suffisante.

Ce point de physiologie est d'une extrême importance, car, si un animal donné avait dû présenter des oscillations eonsidérables dans les richesses moyennes de ses diverses lactations, être bon beurrier à l'une, moińs bon à une autre, médiocre à une troisième, toute base sérieuse de sélection reposant sur le rendement en matière grasse échappait, puisque la pérennité de la qualité butyreuse disparaissait. Puisque.c'est le contraire qui est vrai, il est done, d'ores et déjà, possible de conclure qu'une vache qui est très butyrogène au cours d'une lactation donnée, le sera aussi dans ses lactations ultérieures. Et comme sa puissance butyrogène est susceptible de se transmettre à sa descendance, elle pourra servir de géniteur, soit par ses filles, soit par ses fils, ear le mâle, plus encore que la femelle, est suseeptible de transmettre les qualités laitières que possédaient ses ascendants femelles.

Mais, si la pesée et le dosage journaliers de la matière grasse du lait ont une valeur indiseutable comme base de la sélection des femelles laitières, un grave obstacle se pose devant leur utilisation régulière, du fait de la grande difficulté pratieque à suivre, jour par jour, traite par traite, les rendements en litres de lait et en grammes de graisse à beurre sur chaque animal. On pouvait done craindre que, dans la pratique, on allait se trouver contraint d'abandonner ce moyen de sélection idéale, à moins que des recherches ne vinssent établir qu'on pouvait substituer au contrôle journalier, laborieux et coûteux, un contrôle périodique dont les épreuves s'échẻlonneraient sur la durée entière de la lactation, et qui pût donner de la valeur laitière et butyrogène d'une femelle une approximation satisfaisante.

Quelle sera la périodicité de ce contrôle? Quelle devra être la durée des épreuves à chaque période de ce contrôle, pour que les chiffres du rendement total annuel obtenus en multipliant les moyennes trouvées par le nombre de jours dans le mois et en additionnant les rendements mensuels ainsi calculés, répondent assez fidèlement_aux résultats que donnerait le contrôle journalier, au point de pouvoir se substituer à eux?

Les uns parlent d'un contrôle de un jour par mois, ou de un jour

LE LAIT, I925 
par quinzaine, de un jour toutes les trois semaines; les autres, d'un eontrôle portant sur deux jours consécutifs par mois, etc.; plusieurs combinaisons sont done offertes, et je répèterai avec MALLÈvre que : "Il nẹ faut pas se dissimuler que ce qu'on gagne alors en commodité, on le perd en sécurité et en exactitude. ») Aussi, cet auteur conseille-t-il d'adopter le contrôle une fois tous les huit ou dix jours.

Derrière la périodicité du contrôle, c'est-à-dire le point de savoir si l'épreuve sera faite tous les mois, toutes les trois semaines, tous les quinze jours, tous les huit ou dix jours, se greffe une autre question : la périodicité étant choisie, quelle sera la durée de l'épreuve? un jour ou deux jours? En combinant les deux données, périodicité et durée, on arrive à se poser une autre question : vaut-il mieux une épreuve mensuelle qui dure deux jours, qu'une épreuve tous les quinze jours, ne durant qu'un jour?

Nous pouvons être guidés dans un pareil choix par les épreuves des concours beurriers. Si nous nous reportons au concours de Rouen, lequel a réuni 88 animaux, nous voyons qu'il peut y avoir des différences assez marquées d'un jour à l'autre dans le taux butyreux moyen du lait de toute la journée. Déjà, lorsque le caleul porte sur l'ensemble des trois traites de la journée, nous voyons disparaître, en quelque sorte, ou du moins s'atténuer fortement, les gros écarts qu'il y a entre les richesses butyreuses de chacune des trois traites; ils viennent se fondre dans une moyenne qui répond mieux à la valeur butyrogène de l'animal. Néanmoins, il y a entre deux jours qui se suivent des différences assez marquées parfois; aussi, en faisant la moyenne des résultats obtenus, pendant ces deux jours, on se rapproche encore davantage de la richesse butyreuse moyenne de la lactation toute entière. Aussi, j'estime qu'il est préférable d'avoir une épreuve qui dure deux jours par mois, que deux épreuves d'un jour tous les quinze jours.

On diminue l'influence du hasard qui pourrait faire décider l'épreuve un jour où la matière grasse du lait subit un fléchissement inexplicable, semblable à celui que nous avons rencontré dans les documents antériẻurs (1).

Trox, dans son étude citée plus loin, confirme les résultats d'ANDERSON et autres auteurs.

Les Associations de contrôle relatent des variations importantes d'un mois à l'autre, sur la base d'une épreuve se ramenant à l'examen, chaque mois, du lait de deux traites qui se suivent. Troy donne 4 tableaux de 6 vaches de races différentes, faisant mention des pourcentages mensuels.

(1) A ce sujet, voir la note de la p. 310. 
TAUX bUtYREUX MENSUELS (SUR DEUX TRAITES PAR MOIS) DU LAIT DE 6 VAChFS RACE HOLSTEIN.

\begin{tabular}{|c|c|c|c|c|c|c|c|c|c|c|c|c|}
\hline Vaches & Mai & Juin & Juillet & Août & Septemb. & Octob. & Noyemb. & Décemb. & Janvier & Févcier & Mars & Avril \\
\hline 1. . & 38,1 & 36 & 51,5 & 57,7 & vêlage & 41,2 & 37 & 39,1 & 43,2 & 40,1 & 39,1 & 9,1 \\
\hline 2. & 41, & 44,3 & 64,9 & 64,9 & & 47,3 & 47,3 & 41,2 & & 46 & 45,3 & 0, \\
\hline 3. & 37,2 & 29 , & 32 & 40 & & 49,5 & 53, & & & 49 & vêlage & 4 \\
\hline 4. & 44,3 & 44,3 & 54 & 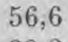 & 43, & 43,2 & 47,4 & & 41 & 41,2 & 42,2 &, 2 \\
\hline & 27,8 & 25,7 & 29,9 & 39,2 & 30,9 & 34 & 40,1 & 48,4 & 48,4 & $"$ & 1) & \\
\hline & 43,2 & 41,2 & 41,2 & 44,3 & 47,4 & 46,3 & vêlage & 41,2 & 41,2 & 50,5 & 45,3 & \\
\hline
\end{tabular}

RACE GUERNESEY.

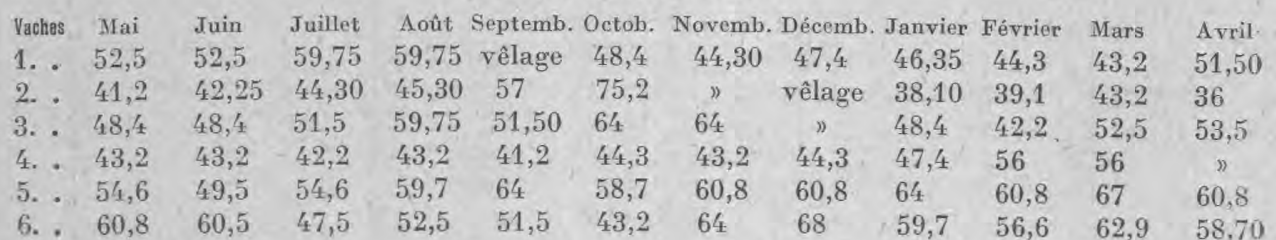

RACE JERSEY.

Vaches Mai Jnin Juillet Août Septemb. Octob. Novemb. Décemb. Janvier Février Mars

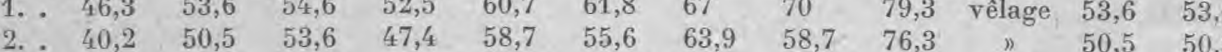

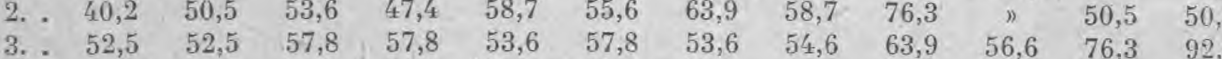

4.. $\begin{array}{lllllllllll}46,3 & 45,3 & 55,6 & 44,3 & 62,8 & 42,2 & 45,3 & 51,5 & 48,4 & 63,9 & \text { ” }\end{array}$

5. . $\begin{array}{llllllllllll}42,2 & 53,6 & 51,5 & 47,4 & 53,6 & 58,7 & 70,9 & 65,9 & 61,8 & 61,8 & 68 & \text { \% }\end{array}$

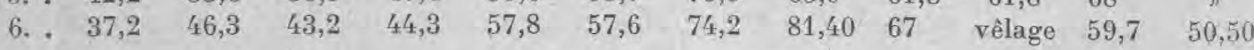

Vaches Mai Juin Juillet Août Septemb. Octob. Novemb. Décemb. Janvier Février Mars

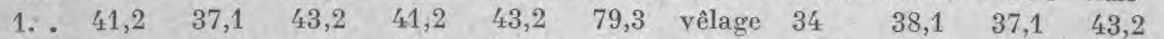

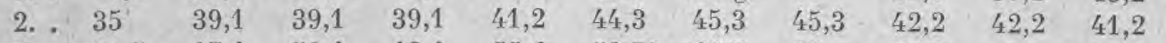

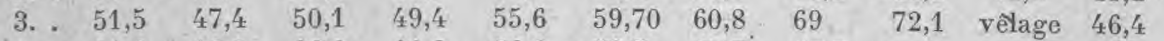

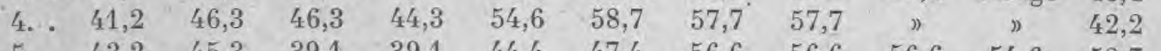

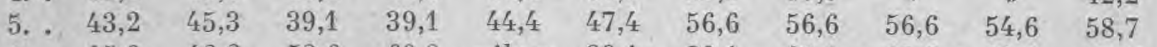

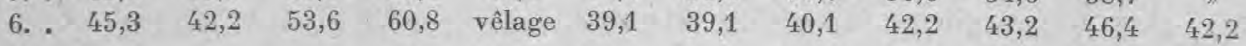

Le graphique suivant (fig. 35), établi sur des documents qui m'ont été communiqués par $M$. Brioux, corrobore les résultats des 4 tableaux de Troy. Il y a de grandes variations d'un mois à l'autre et il est plus que probable que si au contrôle mensuel on substituait le contrôle tous les jours, on aurait des courbes d'une allure plus régulière.

Le graphique de la figure 36 se rapporte à la chèvre. Il est intéressant en ce qu'il s'agit ici d'un même animal suivi pendant cinq lactations consécutives. Evidemment, les cinq courbes sont assez ramassées mais elles n'en ont pas moins chacune une allure propre différente de celles des autres. Un taux butyreux mensuel vraiment moyen, c'est-à-dire établi sur la base des taux de tous les jours du 
Vache 65. Production totale en 10 mois : 4.134 kilogrammes de lait; 189 kilogrammes de matịère grasse.

Vache 66. Production totale en 19 mois : 5.524 kilogrammes de lait; 163 kilogrammes de matière grasse.

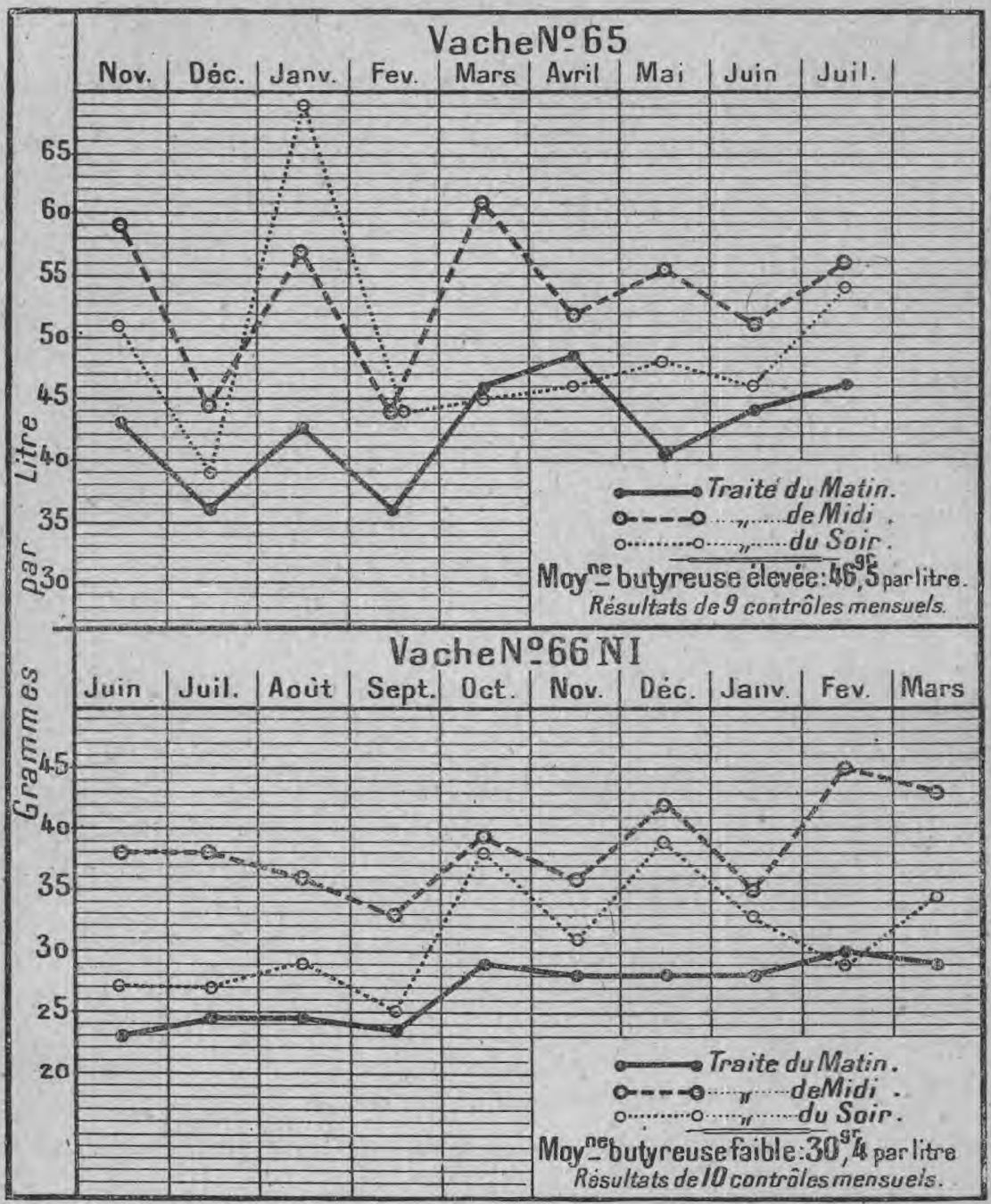

FIG. 35 .

mois, aurait donné des courbes plus régulières et plus serrées l'une près de l'autre.

En dépit des innombrables documents que nous fournit le classement des animaux sur les registres de leur race dans tous les pays 
Taux butyreux mensuel moyen du lait de la même chèvre pendant cinq lactations consécutives. (Institut zootechnique de l'Ecole vétérinaire de Dresde).

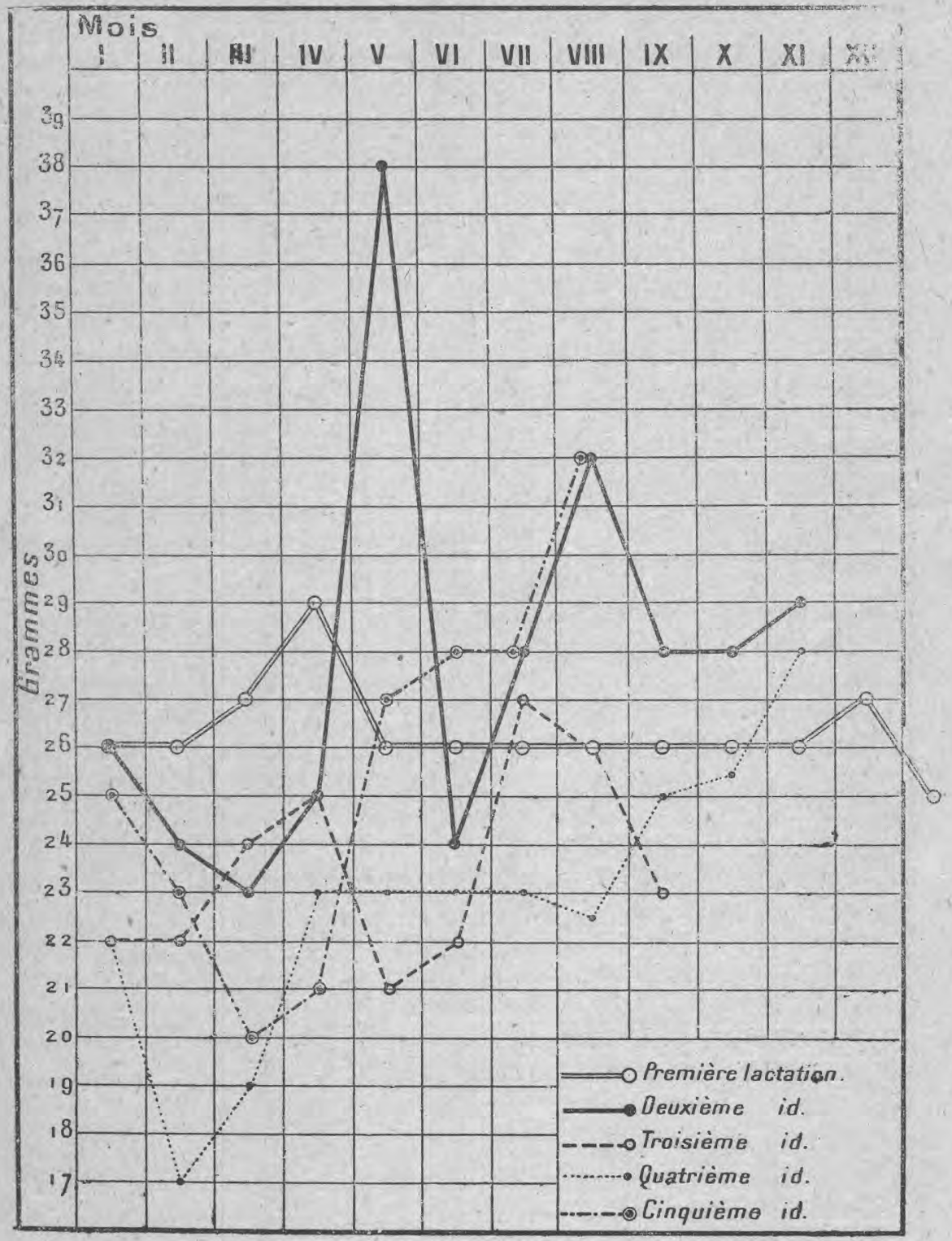

FIG. 36. 
du monde qui se sont orientés vers le contrôle laitier, la question que soulève la périodicité et la durée des épreuves dudit contrôle, n'est pas encore tout à fait résolue, et elle appelle des recherches dans le sens que je viens d'indiquer.

Pour les animaux d'élite ou pour ceux dont la valeur marchande, ou celle de leurs descendants, est considérable, les Amérieains du Nord suivent la technique de Fleischmann et Hittcher; pour les autres de moindre valeur, mais qu'il importe de classer cependant parce que leurs qualités laitières et butyrogènes sont néanmoins remarquables, on procède à des épreuves mensuelles.

Si l'on relit tous les travaux qui ont paru dans le numéro de la revue le Lait auquel je faisais allusion tout à l'heure, on remarquera que la plus grande diversité règne dans le choix de la périodicité et de la durée des épreuses. Cela justifie une fois de plus l'opinion que j'émettais plus haut sur l'intérêt qu'il y aurait à entreprendre systématiquement des recherches dans ce sens. Elles exigeront fatalement un grand nombre d'animaux, car les ferait-on porter sur un petit nombre, il y aurait toujours à craindre l'intervention de fluctuations désordonnées qui pourraient troubler les moyennes réelles à rechercher.

L'importance de la conservation chez un animal, pendant toute sa carrière laitière, de sa valeur butyrogène moyenne, est mise une fois de plus en relief dans le travail de Maynard et Myers. Ils ont rassemblé dans des courbes la production en lait et en matière grasse d'un assez grand nombre de femelles laitières au cours de toutes leurs lactations et ils ont notamment eonstaté que e'était à la huitième lactation que la production, tant du lait que de la matière grasse, était maxima, c'est-à-dire de la neuvième à la dixième année de leur âge. Ces graphiques, nous les reproduisons dans les fig. 37 et 38 . Ils montrent un parallélisme très frappant de l'ensemble des deux courbes, lequel traduit, en somme, l'observation capitale qui ressort des recherches de Freischmann et Hrttcher : c'est que la teneur moyenne annuelle en matière grasse ne varie pas au cours des différentes lactations. La valeur de la documentation de-Maynard et Myers résulte du dépouillement de recherches datant de trente ans, portant sur près de 400 animaux de toutes races, et qui sont dues à M. H.-H. Wing.

S'il y a plus de lait produit, il y a, évidemment, plus de matière grasse produite, pondéralement parlant, et la moyenne, c'est-à-dire le quotient de la quantité de matière grasse par la quantité de lait, varie fort peu. "D'une lactation à l'autre, nous dit MALLÈvre, la différence de richesse butyreuse ne dépasse pas quelques grammes 
- ordinairement 1 à 2 gr. par litre - à la condition sous-entendue que la santé reste bonne et la nourriture suffisante. ")

" Il convient d'ajouter néanmoins, dit-il encore, que la richesse butyreuse moyenne est peut-être un peu plus élevée dans les premières laetations d'un animal s. Le fait, s'il existe réellement,'a besoin d'être confirmé. Il ne peut s'agir, èn tous eas, que d'une supériorité très faible, à peine sensible, en faveur des premières lactations, et ne dépassant pas les proportions indiquées à l'instant de 1 à 2 gr. par litre.

Mais le point essentiel, eneore une fois, qu'il importe de retenir, c'est qu'il n'y a pas de grandes différences d'une lactation à l'autre, et il ne faudrait pas exciper de l'âge avancé d'une vache pour expliquer un taux butyreux faible et persistant, qui serait dû, en réalité, à là fraude.

Faisons remarquer eneore que le maintien du taux butyreux du lait peut aller de-pair avec une augmentation notable de la quantité de lait produit; l'abondance et la richesse peupent donc marcher conjointement, ee qui est d'une importance considérable au point de vue économique.

Mais cette remarque - et il est essentiel de le souligner - né vaut que dans le cadre dessiné par l'utilisation du eontrôle laitier, en vue de l'amélioration de la production du lait en quantité et en qualité. Elle vaut moins, lorsque nous considérons un animal quelconque isolé et qui peut avoir des sautes brusques dans la production de son lait, une augmentation ou une diminution pouvant tenir à un changement de régime : passage du vert au sec ou inversement, passage de la vie à l'étable à la vie au plein air ou inversement encore.

Dans ce eas, la plupart des auteurs s'aceordent à dire qu'une augmentation marquée du lait peut être suivie d'une baisse dans le taux butyreux. Nous verrons ce qu'il faut en penser lorsque nous étudierons le lait de mélange. Iei encore, c'est le grand nombre des animaux en expérience qui permettra de conclure; le faire sur un seul individu, c'est risquer de mal interpréter des chiffres pris au hasard.

Toutefois, le graphique ci-joint, fig. 39 , dressé d'après le travail de Buckley nous montre que le passage du sec au vert ne détermine pas de différence dans le taux butyreux du lait. Les irrégularités de la courbe sont de même amplitude à droite qu'à gauche. Notons que l'animal était bien nourri lors du régime d'étable.

L'influence de la race sur la richesse butyreuse du lait. - L'importance du contrôle laitier n'a pas besoin d'être 
La production du lait en relation avec le nombre de lactations.

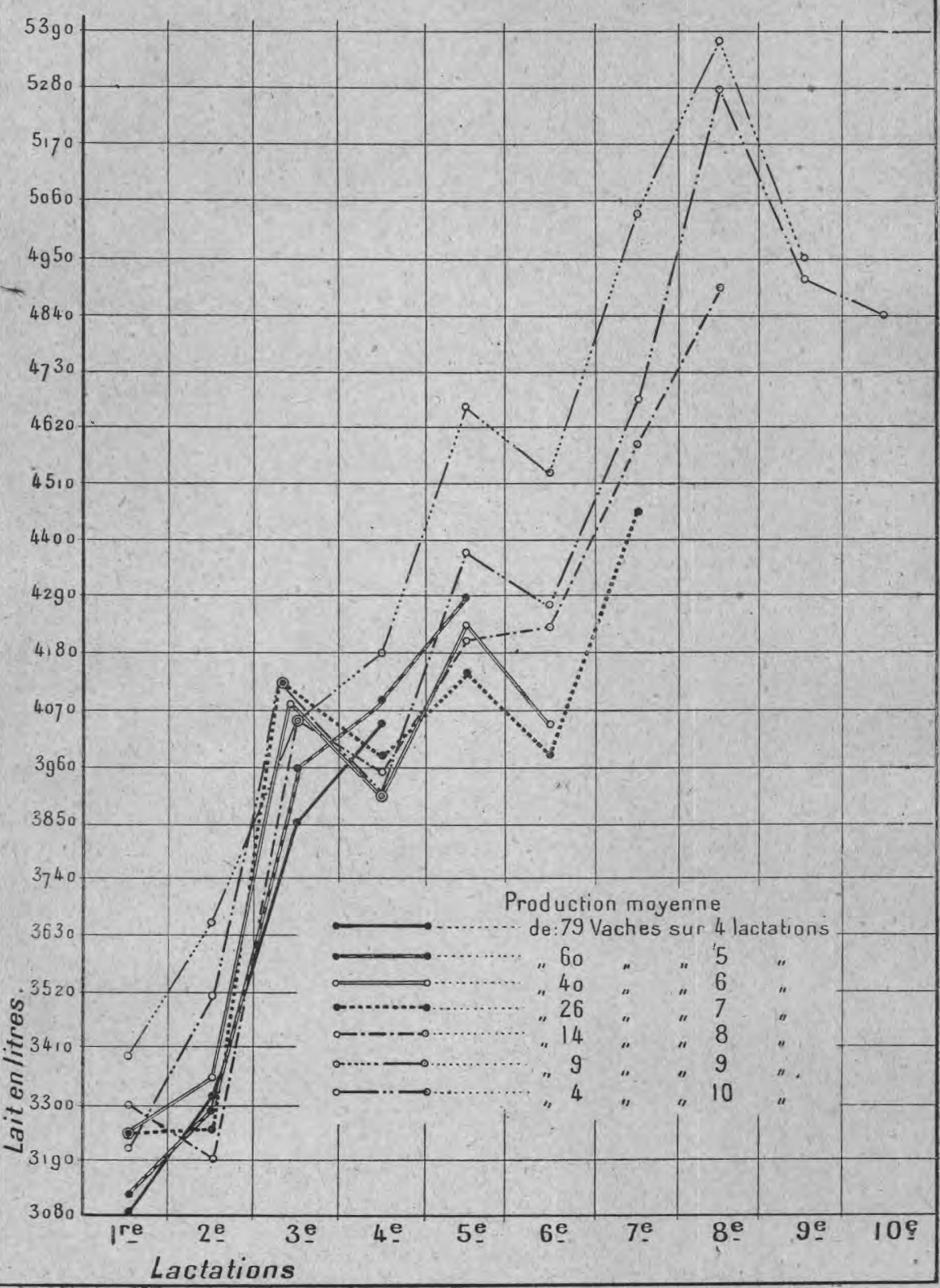

FIG. 37 . 


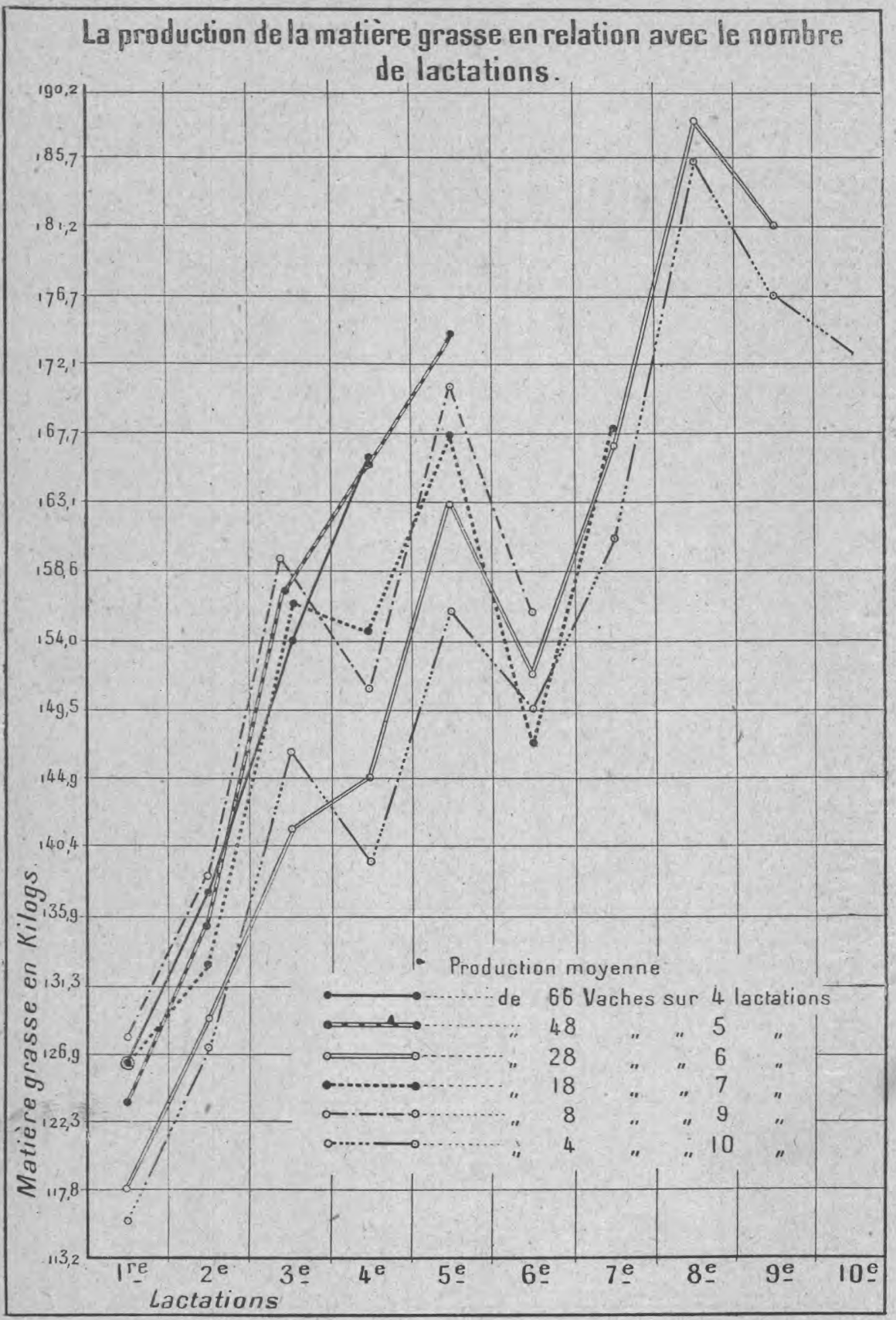

FIG. 38. 
COURBE OU TAUX BUTYREUX OU LAIT D'UNE VACHE AYANT VÉLE LE 20 JANVIER ET TRAITE A $6 H^{\prime \prime}$ "DU MATIN ET A 3H"OE L'APRESS-MIDI

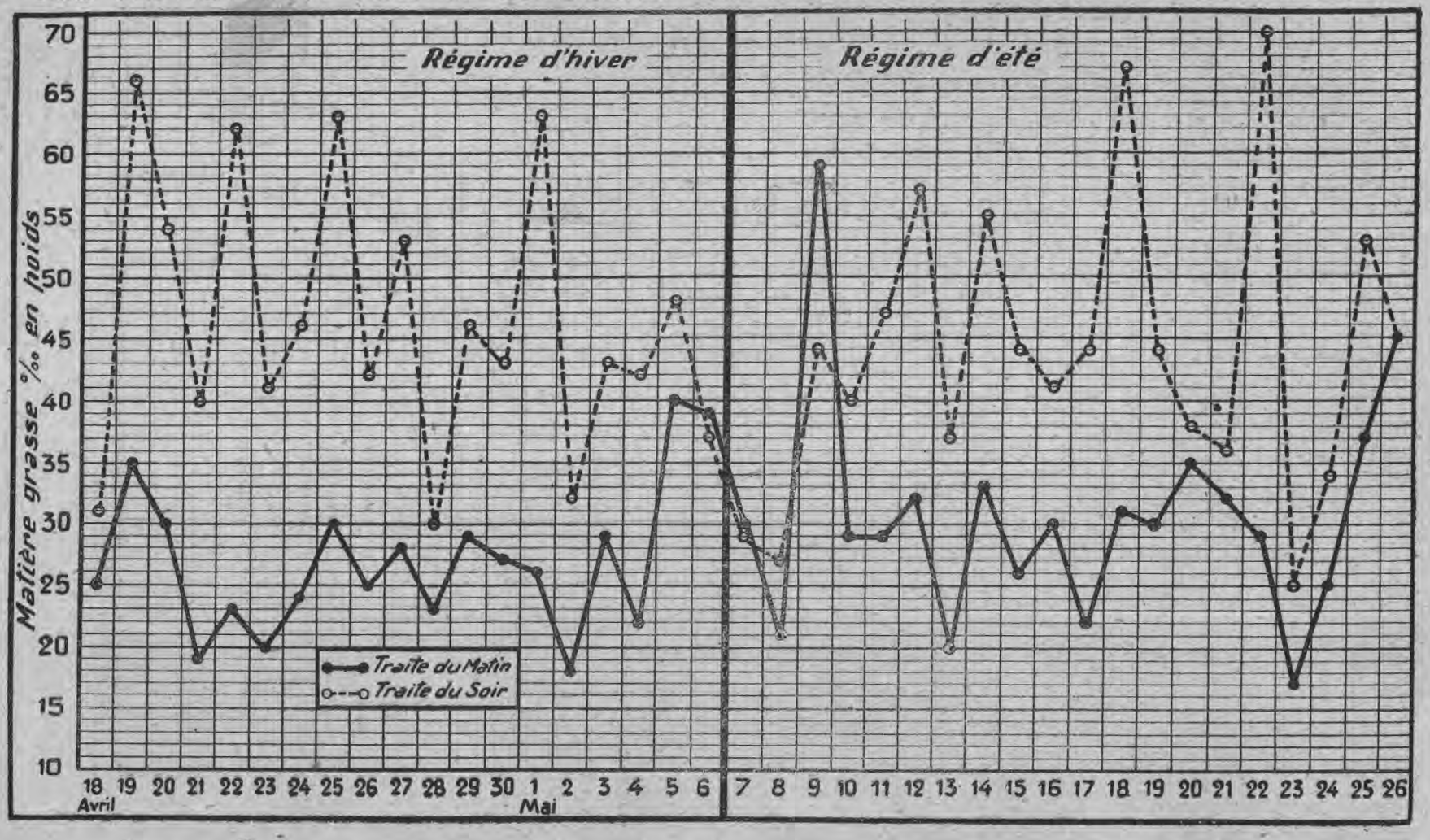

Fig, 39. 
relevée une fois de plus ici, mais nous eroyons utile, néanmoins, de donner les deux graphiques ei-joints (fig. 40 et 41).

Dans la figure 40 , on apprécie l'influence de la race sur la richesse butyreuse du lait; un pareil graphique avait été donné autrefois par Mallèvre, mais j'ai pensé qu'il y avait peut-être lieu

INFLUENCE DE LA RACE SUR LA RICHESSE DU LAIT EN MATIERE GRASSE

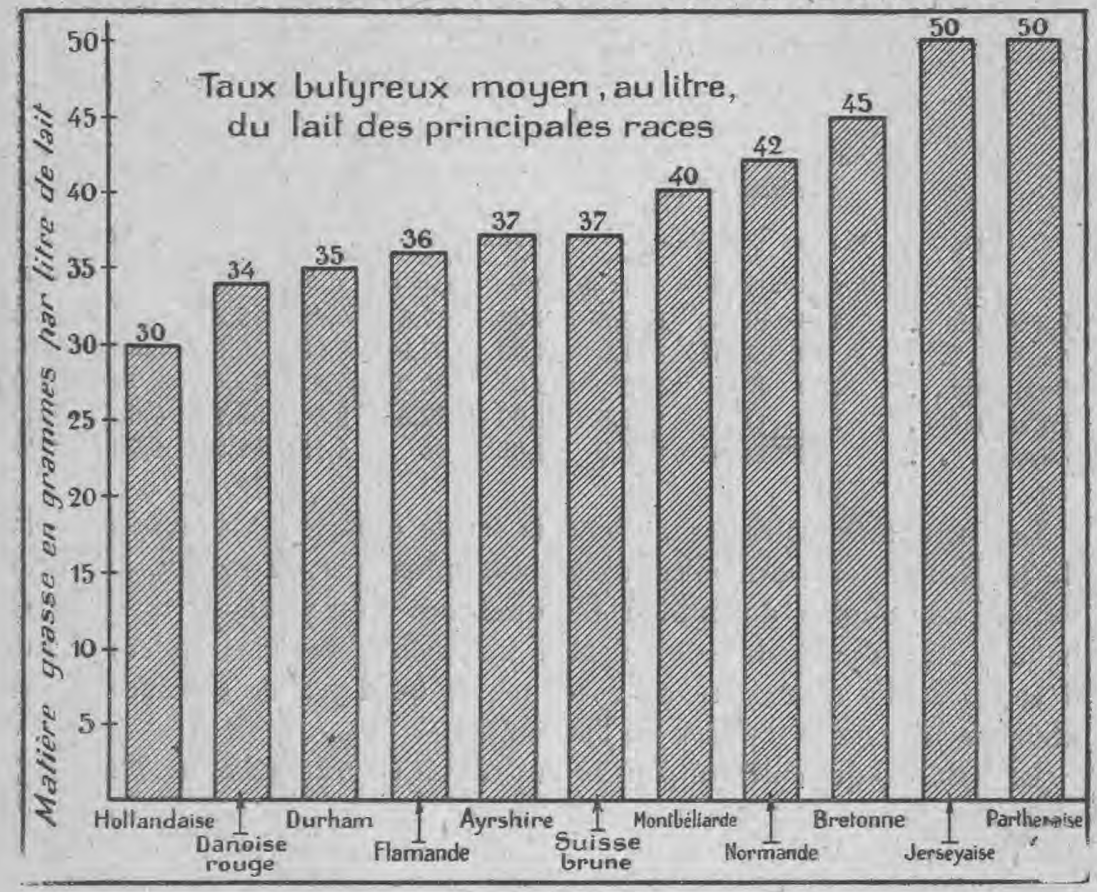

FiG. 40.

de le complèter et d'en corriger quelques données. Celles qui sont présentées iei ne sont peut être pas elles-mêmes à l'abri de la critique, car, en y réfléchissant bien, on constate que, pour rendre un semblable doeument valable, il faut accumuler une quantité considérable de données analytiques, les dépouiller avee discernement et en faire une moyenre. Or, un pareil travail est plus compliqué qu'il ne semble à première vue et la recherche des sources est parfois difficile. Pour fixer la moyenne de la race normande, j'ai pu disposer des chiffres intéressants apportés par M. Brioux et de la figure 41 qui suit on peut, en effet, sans la crainte d'une erreur préjudiciable, extraire la moyenne de 42 grammes pour cette race. 
Ce graphique met en relief le fait bien connu que la richesse du lait en matière grasse varie avec la race. La Parthenaise et la Jerseyaise sont éminemment butyrogènes; la Hollandaise est celle qui occupe le bas de l'échelle. Le tableau de H. Boucher, professeur de zootechnie à l'Ecole vétérinaire de Lyon, tableau qui parut à l'Exposition d'Hygiène urbaine de Lyon en 1914 (Section de laiterie), celui qui a été publié par le Laboratoire municipal de Paris, donnent des chiffres parfois assez différents de ceux du graphique, tant il est vrai qu'il est difficile, même icì, d'avoir quelque concordance.

COMPOSITION MOYENNE DU LAIT DE CERTAINES RACES

(Analyses faites au Laboratoire municipal de Paris sur des échantillons d'origine certaine (1).

\begin{tabular}{|c|c|c|c|c|c|c|c|c|c|c|}
\hline \multirow{2}{*}{$\begin{array}{c}\text { Races } \\
-\end{array}$} & \multirow{2}{*}{\multicolumn{2}{|c|}{$\begin{array}{l}\text { Nombre } \\
\text { de vaches }\end{array}$}} & \multicolumn{2}{|c|}{ Eau } & \multicolumn{2}{|c|}{ Extrait sec } & \multicolumn{2}{|c|}{ Matière grasse } & \multicolumn{2}{|c|}{ Lactose (4) } \\
\hline & & & & & $0 / 0$ & au litre & $0 / 0$ & au litre & $0 / 0$ & au litre \\
\hline & & 176 & & & & & & & & \\
\hline & & 69 & & & & & & & & \\
\hline & & 200 & & & & & & & & \\
\hline & & 350 & & & & & & & & \\
\hline isse & & 56 & 86,91 & 896,9 & 13,09 & 135 & 4,15 & 42,75 & 4,73 & 48,70 \\
\hline
\end{tabular}

Les fraudeurs connaissent bien la faible qualité butyreuse du lait de la Hollandaise au point d'en jouer plus que de raison, car une seule Hollandaise au milieu de quelques autres races différentes peut suffire, disent-ils - et c'est là un raisonnement simpliste, dont il serait facile de mettre en évidence ce qu'il y a de spécieux - à abaisser le taux butyreux de l'ensemble.

Le tableau qui suit, emprunté au travail de DE BRÉvans, (loc. cit.) montre qu'il ne faut rien exagérer. Il est vrai qu'il s'agissait de bêtes de nourrisseurs, en général très belles et bien choisies.

Le graphique de la fig. 40 appelle une observation digne d'être notée, et qui rentre dans le cadre-de celle que j'ai faite plus haut au sujet des recherehes de Fleischmann et Hittcher : Quand un animal d'une race donnée quitte l'habitat usuel de celle-ci, défini par la région où il vit et le climat, il peut arriver qu'il dégénère, en quelque sorte; dans ce cas, c'est la quantité de lait produit qui est atteinte, ce n'est pas le taux butyreux moyen; en se transplan-

(1) Je n'ai gardé du tableáu que les indications relatives à un nombre important d'animaux.

(2) Il s'agit de pourcentages pour 100 grammes de lait.

(3) Chiffres ramenés au litre.

(4) Je n'ai pas retenu les chiffres de la caséine et des cendres, car comme l'analyse " ferme », ces deux données ne sauraient représenter le poids des matières minérales, ni celui de la caséine déterminée par différence. 


\section{Traites effectuées à Paris, en présence des inspecteurs. du Laboratoire munioipal.}

(Mars, Avril, Mai $188_{2}$ )

\begin{tabular}{|c|c|c|c|c|}
\hline $\begin{array}{c}\text { NombRe } \\
\text { de } \\
\text { Vaches }\end{array}$ & RACES & TRAITE & $\begin{array}{l}\text { MATIËRE } \\
\text { ORASSE } \\
\text { pour } 1 \mathrm{~kg} \\
\text { de lait. }\end{array}$ & 7. ALIMIENTATION \\
\hline 23 & 3 Hollandaises, 20 Normandes & malin & 39,2 & Drêche, herbe, paille. \\
\hline 15 & 15 Hollandaises ...... & - & 38,2 & Betteraves, son, paille, foin \\
\hline 2 & 2 Hollandaises . . , . . . & - & 41,8 & Betteraves, drêche, foin, paille. \\
\hline 2 & 2 Flamandes . . . . . & - & 43,2 & Drèche, paille, son. \\
\hline 13 & 11 Hollandaises, 2 Flamandes. & - & 40,6 & Drèche, betteraves, paille, foin. \\
\hline $\begin{array}{l}26 \\
26\end{array}$ & $\begin{array}{l}\text { Flamandes, Hollandaises, Pic. } \\
\text { Flamandes, Hollandaises, Pic. }\end{array}$ & E & $\begin{array}{l}41,6 \\
39,2\end{array}$ & $\begin{array}{l}\text { Drêche, betteraves, paille, foin. } \\
\text { Drêche, betteraves, paille, foin. }\end{array}$ \\
\hline $\begin{array}{r}20 \\
4\end{array}$ & 2 Hollandaises, 1 Pic,, 1 Flam. & -1 & 34,6 & $\begin{array}{l}\text { Oreche, betteraves, palle, foin. } \\
\text { Drêche, foin, son, féveroles. }\end{array}$ \\
\hline 12 & Normandes, Flam., Holl... . & - & 44 & $\begin{array}{l}\text { Féverolles, remoulage, foin, } \\
\text { paille. }\end{array}$ \\
\hline 3 & 2 Hollandaises, 1 Normande. & 一 & 36,9 & Drêche, son, betteraves, foin. \\
\hline 11 & 8 Hollandaises, 3 , Flamandes . & - & 38,1 & $\begin{array}{l}\text { Son, earottes, luzerne, féve- } \\
\text { rolles, foin. }\end{array}$ \\
\hline 21 & 19 Hollandaises, 2 Flamandes . & - & 36,9 & $\begin{array}{l}\text { Drêche, son, cosses de fève, } \\
\text { paille, foin. }\end{array}$ \\
\hline 26 & 18 Hollandaises, 8 Flamandes . & 一 & 33,4 & Drêche, betteraves, paille, son. \\
\hline 20 & 6 Hollandaises, 6 Pic., 8 Flam. & - & 47,4 & Drêche fiquide, son, foin, paille. \\
\hline 20 & 6 Hollandaises, 6 Pic., 8 Flam. & - & 39,2 & Drèche liquide, son, foin, paille. \\
\hline 20 & 6 Hollandaises, 6 Pic., 8 Flam. & - & $\begin{array}{l}36,9 \\
38,1\end{array}$ & Drêche liquide, son soin, paille. \\
\hline 22 & Hollandaises.......... & - & 38,1 & $\begin{array}{l}\text { Son, brisures de fèves, paille, } \\
\text { foin. }\end{array}$ \\
\hline 25 & 15 Hollandaises, 10 Flamandes. & $=$ & 36,9 & $\begin{array}{l}\text { Betteraves, foin, remoulage, } \\
\text { luzerne, son. }\end{array}$ \\
\hline 18 & 8 Hollandaises, 10 Flamandes. & - & 41,6 & $\begin{array}{l}\text { Betteraves, foin, remoulage, } \\
\text { luzerne, son. }\end{array}$ \\
\hline 18 & 10 Flamandes, 8 Hollandaises. & - & 42,7 & $\begin{array}{l}\text { Betteraves, son, pommes de } \\
\text { terre. }\end{array}$ \\
\hline 18 & 8 Hollandaises, 3 Pic., 7 Flam. & 一 & 38,1 & Drêche liquide, son, foin, paille. \\
\hline 24 & 12 Hollandaises, 6 Pic., 6 Norm. & - & $\begin{array}{l}38,1 \\
489\end{array}$ & Drêche, pulpe, son, foin, paille. \\
\hline $\begin{array}{l}10 \\
22\end{array}$ & 19 Hollandaises, 3 Normandes. & - & $\begin{array}{l}48,9 \\
36,9\end{array}$ & $\begin{array}{l}\text { Son, betteraves, foin, paille. } \\
\text { Son, betteraves, foin, paille. }\end{array}$ \\
\hline 17 & 7 Holl., 3 Suisses, 7 Flam.. & - & 32,2 & $\begin{array}{l}\text { Drêche, pulpe, foin, paille, } \\
\text { cosses de fèves. }\end{array}$ \\
\hline 11 & 1 Flam., 4 Suisses, 6 Norm. . & 一 & 40,4 & $\begin{array}{l}\text { Betteraves, fourrage, seigle, } \\
\text { recoupettes. }\end{array}$ \\
\hline $\begin{array}{l}10 \\
22\end{array}$ & $\begin{array}{l}2 \text { Hollandaises, } 8 \text { Picardes. } \\
8 \text { Norm., } 3 \text { Suisses, } 4 \text { Holl, }\end{array}$ & - & 36,9 & Betteraves, son, paille. \\
\hline & 7 Flamandes $\cdot 4$. & & 36,9 & Drêche, betteraves, son, paille. \\
\hline $\begin{array}{l}16 \\
16\end{array}$ & $\begin{array}{l}6 \text { Holl., } 4 \text { Flam., } 2 \text { Pic., } 4 \text { Nor. } \\
6 \text { Holl., } 4 \text { Flam., } 2 \text { Pic., } 4 \text { Norm. }\end{array}$ & - & $\begin{array}{l}40,4 \\
40,4\end{array}$ & Betteraves, maïs, paille, son. \\
\hline 13 & $\begin{array}{l}6 \text { Holl., } 4 \text { Flam., } 2 \text { Pie., } 4 \text { Norm. } \\
\text { Hollandaises. . . . . . . . }\end{array}$ & soir & 34,6 & $\begin{array}{l}\text { Betteraves, maîs, paille, son. } \\
\text { Betteraves, remoulage, son, foin. }\end{array}$ \\
\hline 7 & Hollandaises ....... & - & 35,8 & Betteraves, son, regain. \\
\hline
\end{tabular}

tant, l'animal ne perd pas son "sang butyreux ». C'est une qualité personnelle qu'il a conservée en dépit du changement notable des circumfusa.

Le graphique 41 traduit ce fait également connu que, dans une 
race donnée, il y a des animaux moins beurriers que d'autres. Cette planche donne la richesse moyenne en matière grasse, pour l'ensemble d'une lactation, chez des vaches de race normande soumises au contrôle laitier de la Société d'Elesage du Normand Cauchois. " La richesse moyenne en matière grasse des laits individuels, chez des vaches de race normandes, oscille entre 30 et 50 gr. par litre;

\section{Moyennes butyreuses pour l'ensemble d'une lactation chez des vaches de race normande.}

(Contrôle laitier de la Société d'élevage du Normand-Cauchois).

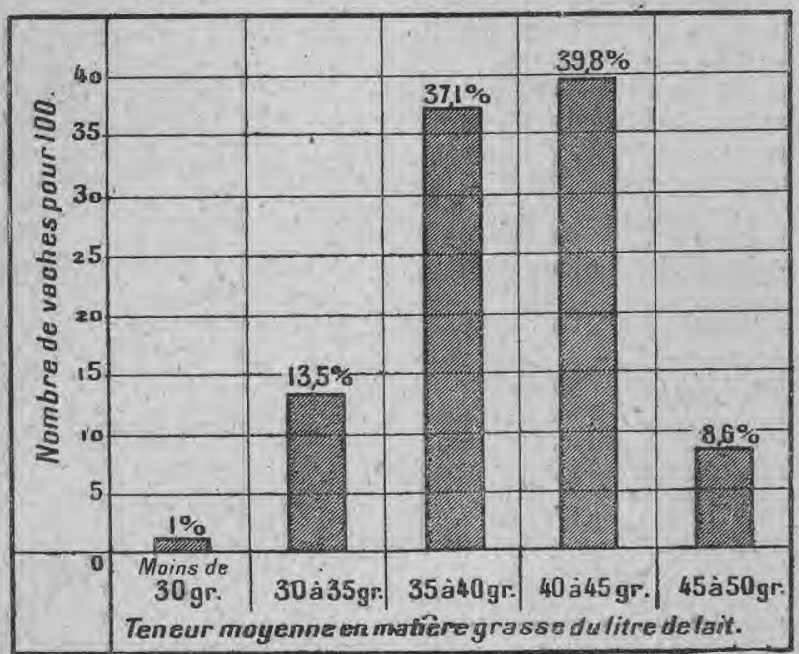

Fis. $4 x$.

mais $77,9 \%$ des vaches contrôlées donne un lait renfermant entre 35 et 45 gr. de matière grasse par litre, pour l'ensemble de leur lactation. ") (Ch. Brioux).

Le contrôle laitier a pour but, d'une part, d'éliminer les animaux qui donnent moins et, d'autre part, de se rapprocher des animaux qui donnent davantage. Il en résultera donc une amélioration de la richesse moyenne dans la race tout entière et très rapidement, en peu d'années, si le contrôle laitier pouvait s'étendre à l'ensemble des animaux de la race normande, les $77,8 \%$ de $M$. Brioux atteindraient presque $100 \%$.

La matière grasse et les laits de mélange. - La variabilité du taux butyreux du lait chez les femelles laitières devant être aceeptée eomme un fait indiscutable, comment s'expliquer cette tendance du plus grand nombre à parler si aisément, et dans les circonstances les plus variées, les plus dissemblables, d'une richeses 
moyenne en matière grasse que l'on cristallise, en quelque sorte, et qui, devenue ainsi véritable étalon, sert de comparaison pour les résultats d'analyses?

Il est facile de répondre à cette question : Si les chifires bas sont fréquemment trouvés, la matin surtout, dans les laits individuels et c'est là un fait qui frappe nettement dans tous les documents sur la matière - il est assez rare que les circonstances nous offrent uniquement le lait de la traite du matin d'un seul animal. Il y a souvent mélange et, dès l'instant qu'il y a mélange, ón peut supposer, a priori, sans crainte de se tromper beaucoup, que les oscillations des courbes butyreuses se neutralisent dans une certaine mesure. Il y a bien des manières de faire des mélanges de laits, et c'est à les examiner avec toutes les conséquences qu'elles comportent que je vais m'emplover.

\section{Le mélange des traites journalières d'un même animal.}

- Nous avons déjà vu plus haut que si nous mélangeons les traites d'un même animal, les trois, lorsqu'il s'agit d'un concours beurrier, les deux, dans les circonstances les plus courantes - mais l'observation vaut aussi bien pour deux que pour trois - le taux souvent assez faible du matin se trouve déjà amorti, sinon neutralisé, en quelque sorte, par le taux plus élevé du soir, quand il y a deux traites, et aussi quand il y en a trois, par celui du midi encore plus élevé que eelui du soir.

\section{Le véritable lait moyen d'une lactation entière.}

J'ajouterai mếme, pour rendre ma pensée plus nette, que si, par hypothèse, on pouvait dans la même cuve rassembler tout le lait d'une lactation entière du même animal, l'éehantillon prélevé serait vraiment le lait moyen, et sa teneur en matière grasse serait la teneur butyreuse moyenne de ladite lactation, et, par extension, celle de l'animal au cours de sa carrière. C'est autour de eette donnée moyenne que viendraient se groupèr tous les chiffres des traites de la lactation entière dont les extrêmes pourraient en être très éloignés.

La moyenne journalière du lait d'un animal donné tend done à se rapprocher de la moyenne de la lactation entière aver laquelle elle peut même se cenfondre parfois, souvent même.

\section{Le mélange des traites de deux jours qui se suivent. -}

Si nous restons toujours dans le cas d'un seul animal, nous pouvons. faire des mélanges plus importants de ses propres traites. Nous pourrons déterminer la richesse butyreuse moyenne de deux jours qui se suivent; e'est ce qui se fait dans les concours laitiers et beurriers, également dans certain mode d'épreuve du contrôle laitier. Comme je le faisais remarquer plus haut, la moyenne de deux jours 
qui se suivent, a plus de chance de se rapprocher de la moyenne butyreuse vraie de la lactation entière que la moyenne d'un seul jour donné.

L'épreuve de sept jours. - Nous pourrons eneore déterminer la richesse moyenne de sept jours qui se suivent. C'est ce qui se fait dans les épreuves dites de sept jours, très employées aux EtatsUnis, soit quelques jours après le vêlage, soit au bout de huit mois. Elles ont pour but, lorsqu'il s'agit d'épreuves après le vêlage, de montrer quelle peut être la puissance de productivité d'une femelle laitière; quand elles sont faites au huitième mois de la lactation et qu'elles sont favorables, elles montrent de plus que la puissance de productivité s'est maintenue. Il est certain que les épreuves de sept jours tous les mois donneraient une image de la valeur de la bête laitière, qui viendrait, vraisemblablement, se calquer presque sur celle qu'on obtiendrait avec le prélèvement journalier.

\title{
(A suipre)
}

\section{CE QUE DOIT SAVOIR UN BON CONTROLEUR. LAITIER ET BEURRIER}

\author{
par A.-M. LEROY \\ Ingénieur agronome, \\ Chef de travaux à l'Institut National Agronomique. \\ - SUITE - \\ TROISIÈME PARTIE \\ UTILILISATION DES RÉSULTATS \\ FOURNIS PAR LE CONTROLE LAITIER ET BEURRIER \\ A LA SÉLECTION DES VACHES LAITIĖRES. \\ Calcul de la production d'une vache au cours d'une lactation donnée. - \\ Influence de l'âge, de l'époque du vélage, de la gestation, du repos \\ de la mamelle, sur la production du lait et du beurre. - Rôle du \\ taureau dans la transmission des caractères laitiers et beurriers et \\ choix des reproducteurs bons raceurs. - Les livres zootechniques : \\ leur utilité, leur organisation, leur tenue. - Rapport entre les livres \\ zootechniques et les herd-books.
}

\section{T. Caleni de la production d'ume vache au cours d'une lactation donmée.}

Pour pouvoir comparer les valeurs productives relatives d'un nombre déterminé de vaches laitières, il importe de trouver une commúne mesure des quantités de lait et de beurre que chacune 